\title{
Differential equations satisfied by modular forms of level 5
}

\author{
By Kazuhide Matsuda \\ Faculty of Fundamental Science, National Institute of Technology, Niihama College, \\ 7-1 Yagumo-chou, Niihama, Ehime 792-8580, Japan \\ E-mail: matsuda@sci.niihama-nct.ac.jp \\ Fax: 81-0897-37-7809
}

\begin{abstract}
This paper describes the derivation of the level 5 versions of Ramanujan's system of ordinary differential equations satisfied by the Eisenstein series, $E_{2}(q), E_{4}(q)$, and $E_{6}(q)$.
\end{abstract}

Key Words: theta function; theta derivatives; rational characteristics.

MSC(2010) 14K25; 11E25

\section{Introduction}

Let $\mathbb{N}_{0}$ and $\mathbb{N}$, denote sets of nonnegative and positive integers, respectively. For $k$ and $n \in \mathbb{N}, \sigma_{k}(n)$ is the sum of the $k$-th power of the positive divisors of $n$, and $\sigma_{k}(n)=0$ for $n \in \mathbb{Q} \backslash \mathbb{N}_{0}$.

The upper half plane $\mathbb{H}^{2}$ is defined by $\mathbb{H}^{2}=\{\tau \in \mathbb{C} \mid \Im \tau>0\}$. Throughout this paper, we set $q=\exp (2 \pi i \tau)$ and define the Dedekind eta function as $\eta(\tau)=q^{\frac{1}{24}} \prod_{n=1}^{\infty}\left(1-q^{n}\right)$.

In [4], Darboux studied a problem in mechanics and differential geometry, and encountered the system of ordinary differential equations (ODEs),

$$
x \frac{d}{d x}\left(u_{1}+u_{2}\right)=u_{1} u_{2}, x \frac{d}{d x}\left(u_{1}+u_{3}\right)=u_{1} u_{3}, x \frac{d}{d x}\left(u_{2}+u_{3}\right)=u_{2} u_{3}, x=\exp (\pi i \tau) .
$$

In [6, pp. 330], Halphen provided the following solution to the problem studied by Darboux,

$$
u_{1}=1+8 \sum_{n=1}^{\infty} \frac{x^{2 n}}{\left(1+x^{2 n}\right)^{2}}, u_{2}=-8 \sum_{n=1}^{\infty} \frac{x^{2 n-1}}{\left(1-x^{2 n-1}\right)^{2}}, u_{3}=8 \sum_{n=1}^{\infty} \frac{x^{2 n-1}}{\left(1+x^{2 n-1}\right)^{2}} .
$$

Equation (1.1) is called Halphen's system.

For studying Painlevé type equations of the third order, Chazy [2] considered the nonlinear differential equation for the complex function

$$
y^{\prime \prime \prime}=2 y y^{\prime \prime}-3\left(y^{\prime}\right)^{2} .
$$


Note that $y=u_{1}+u_{2}+u_{3}=\pi i E_{2}(\tau)$ is a solution of Chazy's equation (1.2), where the Eisenstein series $E_{2}, E_{4}$, and $E_{6}$ are respectively defined by

$$
\begin{aligned}
& E_{2}(q)=E_{2}(\tau):=1-24 \sum_{n=1}^{\infty} \sigma_{1}(n) q^{n}, E_{4}(q)=E_{4}(\tau):=1+240 \sum_{n=1}^{\infty} \sigma_{3}(n) q^{n} \\
& E_{6}(q)=E_{4}(\tau):=1-504 \sum_{n=1}^{\infty} \sigma_{5}(n) q^{n} .
\end{aligned}
$$

In [11, Ramanujan derived the following system of ODEs,

$$
q \frac{d E_{2}}{d q}=\frac{\left(E_{2}\right)^{2}-E_{4}}{12}, q \frac{d E_{4}}{d q}=\frac{E_{2} E_{4}-E_{6}}{3}, q \frac{d E_{6}}{d q}=\frac{E_{2} E_{6}-\left(E_{4}\right)^{2}}{2} .
$$

In particular, by eliminating $E_{4}$ and $E_{6}, y(\tau)=\pi i E_{2}(\tau)$ is observed to be a solution to Chazy's equation (1.2). Further, Ohyama [10] showed that Halphen's differential field is an extension of Ramanujan's differential field, whose Galois group is the symmetric group $S_{3}$.

In [8] and [9], ODEs satisfied by the cubic theta functions were derived as

$$
\begin{aligned}
& a(q)=\sum_{m, n \in \mathbb{Z}} q^{m^{2}+m n+n^{2}}, b(q)=\sum_{m, n \in \mathbb{Z}} \omega^{n-m} q^{m^{2}+m n+n^{2}}, \\
& c(q)=\sum_{m, n \in \mathbb{Z}} q^{\left(n+\frac{1}{3}\right)^{2}+\left(n+\frac{1}{3}\right)\left(m+\frac{1}{3}\right)+\left(m+\frac{1}{3}\right)^{2}}, \omega=e^{\frac{2 \pi i}{3}},|q|<1 .
\end{aligned}
$$

Therefore, Huber used Ramanujan's theory of theta functions. In addition, Cooper [3] also treated ODEs satisfied by modular forms by using Ramanujan's theory. In the present study, the theory of theta functions with rational characteristics has been adopted. Noted is that Hermite [7] introduced theta functions with characteristics, and Farkas and Kra [5] developed the theory of theta functions with rational characteristics.

The aim of the current research was to derive the ODEs satisfied by the modular forms of level 5, and to obtain an extension of Ramanujan's differential field by using the modular forms of level 5 .

The main theorems of this study are as follows. 
Theorem 1.1. For $q \in \mathbb{C}$ with $|q|<1$, set

$$
\begin{aligned}
& P(q)=\cot \frac{2 \pi}{5}+4 \sin \frac{4 \pi}{5} \sum_{n=1}^{\infty}\left(d_{1,5}(n)-d_{4,5}(n)\right) q^{n}-4 \sin \frac{2 \pi}{5} \sum_{n=1}^{\infty}\left(d_{2,5}(n)-d_{3,5}(n)\right) q^{n} \\
& Q(q)=\cot \frac{\pi}{5}+4 \sin \frac{2 \pi}{5} \sum_{n=1}^{\infty}\left(d_{1,5}(n)-d_{4,5}(n)\right) q^{n}+4 \sin \frac{4 \pi}{5} \sum_{n=1}^{\infty}\left(d_{2,5}(n)-d_{3,5}(n)\right) q^{n} \\
& R(q)=E_{2}(q)=1-24 \sum_{n=1}^{\infty} \sigma_{1}(n) q^{n} .
\end{aligned}
$$

Then, we have

$$
\begin{aligned}
q \frac{d}{d q} P & =\frac{-13 P^{3}-39 P^{2} Q+47 P Q^{2}-9 Q^{3}+2 P R}{24}, q \frac{d}{d q} Q=\frac{9 P^{3}+47 P^{2} Q+39 P Q^{2}-13 Q^{3}+2 Q R}{24} \\
q \frac{d}{d q} R & =\frac{5 P^{4}-15 P^{3} Q-155 P^{2} Q^{2}+15 P Q^{3}+5 Q^{4}+R^{2}}{12} .
\end{aligned}
$$

Theorem 1.2. Further, for $q \in \mathbb{C}$ with $|q|<1$, set

$$
\begin{aligned}
& P(q)=1+10 \sum_{n=1}^{\infty}\left(d_{2,5}(n)-d_{3,5}(n)\right) q^{n}, Q(q)=3+10 \sum_{n=1}^{\infty}\left(d_{1,5}(n)-d_{4,5}(n)\right) q^{n}, \\
& R(q)=E_{2}\left(q^{5}\right)=1-24 \sum_{n=1}^{\infty} \sigma_{1}(n) q^{5 n} .
\end{aligned}
$$

Then, we have

$$
\begin{aligned}
q \frac{d}{d q} P & =\frac{13 P^{3}+39 P^{2} Q-47 P Q^{2}+9 Q^{3}+50 P R}{120}, q \frac{d}{d q} Q=\frac{-9 P^{3}-47 P^{2} Q-39 P Q^{2}+13 Q^{3}+50 Q R}{120}, \\
q \frac{d}{d q} R & =\frac{P^{4}-3 P^{3} Q-31 P^{2} Q^{2}+3 P Q^{3}+Q^{4}+125 R^{2}}{300} .
\end{aligned}
$$

The remainder of this paper is organized as follows. Section 2 provides an overview of Farkas' and Kra's theory of theta functions with rational characteristics. Next, Section 3 discusses Weierstrass elliptic function theory, and Section 4 describes the preliminary results obtained for $P, Q$, and $R$. Further, Section 5 derives the identities of the theta derivatives. Sections 6 and 7 provide the proofs for Theorems 1.1 and 1.2, respectively. Section 8 presents the eta products, $\eta^{5}(\tau) / \eta(5 \tau)$ and $\eta^{5}(5 \tau) / \eta(\tau)$. Section 9 discusses the theorem of Farkas and Kra stating the theta derivatives. Finally, Section 10 derives the Riccati equations satisfied by the modular forms of level 5 . 


\section{Acknowledgments}

This work was supported by JSPS KAKENHI Grant Number JP17K14213.

\section{Properties of the theta functions}

\subsection{Definitions}

Following the work of Farkas and Kra [5], we introduce the theta function with characteristics, which is defined by

$$
\theta\left[\begin{array}{c}
\epsilon \\
\epsilon^{\prime}
\end{array}\right](\zeta, \tau)=\theta\left[\begin{array}{c}
\epsilon \\
\epsilon^{\prime}
\end{array}\right](\zeta):=\sum_{n \in \mathbb{Z}} \exp \left(2 \pi i\left[\frac{1}{2}\left(n+\frac{\epsilon}{2}\right)^{2} \tau+\left(n+\frac{\epsilon}{2}\right)\left(\zeta+\frac{\epsilon^{\prime}}{2}\right)\right]\right)
$$

where $\epsilon, \epsilon^{\prime} \in \mathbb{R}, \zeta \in \mathbb{C}$, and $\tau \in \mathbb{H}^{2}$.

The relation between theta functions with rational characteristics and Jacobi theta functions is given by

$$
\vartheta_{1}(\pi \zeta)=-\theta\left[\begin{array}{l}
1 \\
1
\end{array}\right](\zeta), \vartheta_{2}(\pi \zeta)=\theta\left[\begin{array}{l}
1 \\
0
\end{array}\right](\zeta), \vartheta_{3}(\pi \zeta)=\theta\left[\begin{array}{l}
0 \\
0
\end{array}\right](\zeta), \vartheta_{4}(\pi \zeta)=\theta\left[\begin{array}{l}
0 \\
1
\end{array}\right](\zeta)
$$

The theta constants are given by

$$
\theta\left[\begin{array}{c}
\epsilon \\
\epsilon^{\prime}
\end{array}\right]:=\theta\left[\begin{array}{c}
\epsilon \\
\epsilon^{\prime}
\end{array}\right](0, \tau)
$$

Furthermore, we denote the theta derivatives by

$$
\theta^{\prime}\left[\begin{array}{c}
\epsilon \\
\epsilon^{\prime}
\end{array}\right]:=\left.\frac{\partial}{\partial \zeta} \theta\left[\begin{array}{c}
\epsilon \\
\epsilon^{\prime}
\end{array}\right](\zeta, \tau)\right|_{\zeta=0}, \theta^{\prime \prime}\left[\begin{array}{c}
\epsilon \\
\epsilon^{\prime}
\end{array}\right]:=\left.\frac{\partial^{2}}{\partial \zeta^{2}} \theta\left[\begin{array}{c}
\epsilon \\
\epsilon^{\prime}
\end{array}\right](\zeta, \tau)\right|_{\zeta=0},
$$

and

$\theta^{\prime \prime \prime}\left[\begin{array}{c}\epsilon \\ \epsilon^{\prime}\end{array}\right]:=\left.\frac{\partial^{3}}{\partial \zeta^{3}} \theta\left[\begin{array}{c}\epsilon \\ \epsilon^{\prime}\end{array}\right](\zeta, \tau)\right|_{\zeta=0}, \theta^{(n)}\left[\begin{array}{c}\epsilon \\ \epsilon^{\prime}\end{array}\right]:=\left.\frac{\partial^{n}}{\partial \zeta^{n}} \theta\left[\begin{array}{c}\epsilon \\ \epsilon^{\prime}\end{array}\right](\zeta, \tau)\right|_{\zeta=0}, \quad(n=1,2,3,4, \ldots)$.

In particular, Jacobi's derivative formula is given by

$$
\theta^{\prime}\left[\begin{array}{l}
1 \\
1
\end{array}\right]=-\pi \theta\left[\begin{array}{l}
0 \\
0
\end{array}\right] \theta\left[\begin{array}{l}
1 \\
0
\end{array}\right] \theta\left[\begin{array}{l}
0 \\
1
\end{array}\right] .
$$




\subsection{Basic properties}

First, we note that for $m, n \in \mathbb{Z}$,

$$
\theta\left[\begin{array}{c}
\epsilon \\
\epsilon^{\prime}
\end{array}\right](\zeta+n+m \tau, \tau)=\exp (2 \pi i)\left[\frac{n \epsilon-m \epsilon^{\prime}}{2}-m \zeta-\frac{m^{2} \tau}{2}\right] \theta\left[\begin{array}{c}
\epsilon \\
\epsilon^{\prime}
\end{array}\right](\zeta, \tau),
$$

and

$$
\theta\left[\begin{array}{c}
\epsilon+2 m \\
\epsilon^{\prime}+2 n
\end{array}\right](\zeta, \tau)=\exp (\pi i \epsilon n) \theta\left[\begin{array}{c}
\epsilon \\
\epsilon^{\prime}
\end{array}\right](\zeta, \tau)
$$

Furthermore, it is easy to see that

$$
\theta\left[\begin{array}{l}
-\epsilon \\
-\epsilon^{\prime}
\end{array}\right](\zeta, \tau)=\theta\left[\begin{array}{c}
\epsilon \\
\epsilon^{\prime}
\end{array}\right](-\zeta, \tau) \text { and } \theta^{\prime}\left[\begin{array}{c}
-\epsilon \\
-\epsilon^{\prime}
\end{array}\right](\zeta, \tau)=-\theta^{\prime}\left[\begin{array}{c}
\epsilon \\
\epsilon^{\prime}
\end{array}\right](-\zeta, \tau)
$$

For $m, n \in \mathbb{R}$, we get

$$
\begin{aligned}
& \theta\left[\begin{array}{c}
\epsilon \\
\epsilon^{\prime}
\end{array}\right]\left(\zeta+\frac{n+m \tau}{2}, \tau\right) \\
& =\exp (2 \pi i)\left[-\frac{m \zeta}{2}-\frac{m^{2} \tau}{8}-\frac{m\left(\epsilon^{\prime}+n\right)}{4}\right] \theta\left[\begin{array}{c}
\epsilon+m \\
\epsilon^{\prime}+n
\end{array}\right](\zeta, \tau) .
\end{aligned}
$$

Note that $\theta\left[\begin{array}{c}\epsilon \\ \epsilon^{\prime}\end{array}\right](\zeta, \tau)$ has only one zero in the fundamental parallelogram, and is given by

$$
\zeta=\frac{1-\epsilon}{2} \tau+\frac{1-\epsilon^{\prime}}{2}
$$

\subsection{Jacobi's triple product identity}

All the theta functions have infinite product expansions given by

$$
\begin{aligned}
\theta\left[\begin{array}{c}
\epsilon \\
\epsilon^{\prime}
\end{array}\right](\zeta, \tau)= & \exp \left(\frac{\pi i \epsilon \epsilon^{\prime}}{2}\right) x^{\frac{\epsilon^{2}}{4}} z^{\frac{\epsilon}{2}} \\
& \times \prod_{n=1}^{\infty}\left(1-x^{2 n}\right)\left(1+e^{\pi i \epsilon^{\prime}} x^{2 n-1+\epsilon} z\right)\left(1+e^{-\pi i \epsilon^{\prime}} x^{2 n-1-\epsilon} / z\right)
\end{aligned}
$$

where $x=\exp (\pi i \tau)$ and $z=\exp (2 \pi i \zeta)$. Therefore, according to Jacobi's derivative formula (2.1), it follows that

$$
\theta^{\prime}\left[\begin{array}{l}
1 \\
1
\end{array}\right](0, \tau)=-2 \pi q^{\frac{1}{8}} \prod_{\substack{n=1 \\
5}}^{\infty}\left(1-q^{n}\right)^{3}, q=\exp (2 \pi i \tau)
$$




\subsection{Spaces of $N$-th order $\theta$-functions}

Following the theory by Farkas and Kra [5], we define $\mathcal{F}_{N}\left[\begin{array}{c}\epsilon \\ \epsilon^{\prime}\end{array}\right]$ as the set of all functions $f$ satisfying the two functional equations,

$$
f(\zeta+1)=\exp (\pi i \epsilon) f(\zeta)
$$

and

$$
f(\zeta+\tau)=\exp (-\pi i)\left[\epsilon^{\prime}+2 N \zeta+N \tau\right] f(\zeta), \quad \zeta \in \mathbb{C}, \tau \in \mathbb{H}^{2},
$$

where $N$ is a positive integer and $\left[\begin{array}{c}\epsilon \\ \epsilon^{\prime}\end{array}\right] \in \mathbb{R}^{2}$. This set of functions is called the space of $N$-th order $\theta$-functions with characteristics $\left[\begin{array}{c}\epsilon \\ \epsilon^{\prime}\end{array}\right]$. Note that

$$
\operatorname{dim} \mathcal{F}_{N}\left[\begin{array}{c}
\epsilon \\
\epsilon^{\prime}
\end{array}\right]=N
$$

For the proof, please refer to [5, pp.133].

\subsection{The heat equation}

The theta function satisfies the following heat equation:

$$
\frac{\partial^{2}}{\partial \zeta^{2}} \theta\left[\begin{array}{c}
\epsilon \\
\epsilon^{\prime}
\end{array}\right](\zeta, \tau)=4 \pi i \frac{\partial}{\partial \tau} \theta\left[\begin{array}{c}
\epsilon \\
\epsilon^{\prime}
\end{array}\right](\zeta, \tau) .
$$

\section{Weierstrass elliptic function theory}

In this paper, we introduce Weierstrass $\wp$-function and $\sigma$-function as follows:

$$
\begin{aligned}
& \wp\left(z ; \omega_{1}, \omega_{2}\right)=\wp(z)=\frac{1}{z^{2}}+\sum_{\substack{(m, n) \in \mathbb{Z}^{2} \\
(m, n) \neq(0,0)}}\left(\frac{1}{\left(z-m \omega_{1}-n \omega_{2}\right)^{2}}-\frac{1}{\left(m \omega_{1}+n \omega_{2}\right)^{2}}\right), \\
& \sigma\left(z ; \omega_{1}, \omega_{2}\right)=\sigma(z)=z \prod_{\substack{(m, n) \in \mathbb{Z}^{2} \\
(m, n) \neq(0,0)}}\left(1-\frac{z}{m \omega_{1}+n \omega_{2}}\right) \exp \left(\frac{z}{m \omega_{1}+n \omega_{2}}+\frac{z^{2}}{2\left(m \omega_{1}+n \omega_{2}\right)^{2}}\right),
\end{aligned}
$$

where $z, \omega_{1}, \omega_{2}$ are complex numbers with $\omega_{2} / \omega_{1} \notin \mathbb{R}$. 
The following formula is recalled from the study by Whittaker and Watson [12, pp. 437, 459]:

$$
\begin{aligned}
\wp^{\prime}(z)^{2} & =4 \wp(z)^{3}-g_{2} \wp(z)-g_{3}, \\
g_{2}\left(\omega_{1}, \omega_{2}\right) & =60 G_{4}\left(\omega_{1}, \omega_{2}\right)=60 \sum_{\substack{(m, n) \in \mathbb{Z}^{2} \\
(m, n) \neq(0,0)}} \frac{1}{\left(m \omega_{1}+n \omega_{2}\right)^{4}}, \\
g_{3}\left(\omega_{1}, \omega_{2}\right) & =140 G_{6}\left(\omega_{1}, \omega_{2}\right)=140 \sum_{\substack{(m, n) \in \mathbb{Z}^{2} \\
(m, n) \neq(0,0)}} \frac{1}{\left(m \omega_{1}+n \omega_{2}\right)^{6}},
\end{aligned}
$$

which implies

$$
\wp^{\prime \prime}(z)=6 \wp^{2}(z)-\frac{1}{2} g_{2}
$$

For $k \in \mathbb{N}$, the Eisenstein series is defined by

$$
G_{2 k}(\tau)=G_{2 k}(1, \tau)=\sum_{\substack{(m, n) \in \mathbb{Z}^{2} \\(m, n) \neq(0,0)}} \frac{1}{(m+n \tau)^{2 k}}, \tau \in \mathbb{H}^{2}
$$

Moreover, from [5, pp. 124], we recall

$$
\begin{aligned}
\wp(z ; 1, \tau) & =\frac{1}{3} \frac{\theta^{\prime \prime \prime}\left[\begin{array}{l}
1 \\
1
\end{array}\right]}{\theta^{\prime}\left[\begin{array}{l}
1 \\
1
\end{array}\right]}-\frac{d^{2}}{d z^{2}} \log \theta\left[\begin{array}{l}
1 \\
1
\end{array}\right](z, \tau), \\
\sigma\left(z ; \omega_{1}, \omega_{2}\right) & =\exp \left(\frac{\eta_{1} z^{2}}{2 \omega_{1}}\right) \frac{\omega_{1}}{\theta^{\prime}\left[\begin{array}{l}
1 \\
1
\end{array}\right]} \theta\left[\begin{array}{l}
1 \\
1
\end{array}\right]\left(\frac{z}{\omega_{1}}, \tau\right),
\end{aligned}
$$

where $\omega_{2} / \omega_{1}=\tau \in \mathbb{H}^{2}$. 


\section{Preliminary results}

Proposition 4.1. For every $\tau \in \mathbb{H}^{2}$, we have

$\frac{\theta^{\prime}\left[\begin{array}{c}1 \\ \frac{1}{5}\end{array}\right]}{\theta\left[\begin{array}{c}1 \\ \frac{1}{5}\end{array}\right]}=-\pi\left\{\cot \frac{2 \pi}{5}+4 \sin \frac{4 \pi}{5} \sum_{n=1}^{\infty}\left(d_{1,5}(n)-d_{4,5}(n)\right) q^{n}-4 \sin \frac{2 \pi}{5} \sum_{n=1}^{\infty}\left(d_{2,5}(n)-d_{3,5}(n)\right) q^{n}\right\}$
$\frac{\theta^{\prime}\left[\begin{array}{c}1 \\ \frac{3}{5}\end{array}\right]}{\theta\left[\begin{array}{c}1 \\ \frac{3}{5}\end{array}\right]}=-\pi\left\{\cot \frac{\pi}{5}+4 \sin \frac{2 \pi}{5} \sum_{n=1}^{\infty}\left(d_{1,5}(n)-d_{4,5}(n)\right) q^{n}+4 \sin \frac{4 \pi}{5} \sum_{n=1}^{\infty}\left(d_{2,5}(n)-d_{3,5}(n)\right) q^{n}\right\}$

where $q=\exp (2 \pi i \tau)$.

Proof. The proposition follows from Jacobi's triple product identity (2.5)

Proposition 4.2. For every $\tau \in \mathbb{H}^{2}$, we have

$\frac{\theta^{\prime}\left[\begin{array}{l}\frac{1}{5} \\ 1\end{array}\right]}{\theta\left[\begin{array}{c}\frac{1}{5} \\ 1\end{array}\right]}=\frac{\pi i}{5}\left\{1+10 \sum_{n=1}^{\infty}\left(d_{2,5}(n)-d_{3,5}(n)\right) y^{n}\right\}, \frac{\theta^{\prime}\left[\begin{array}{c}\frac{3}{5} \\ 1\end{array}\right]}{\theta\left[\begin{array}{c}\frac{3}{5} \\ 1\end{array}\right]}=\frac{\pi i}{5}\left\{3+10 \sum_{n=1}^{\infty}\left(d_{1,5}(n)-d_{4,5}(n)\right) y^{n}\right\}$

where $y=\exp (2 \pi i \tau / 5)$.

Proof. The proposition follows from Jacobi's triple product identity (2.5).

Proposition 4.3. For every $\tau \in \mathbb{H}^{2}$, we have

$$
\frac{\theta^{\prime \prime \prime}\left[\begin{array}{l}
1 \\
1
\end{array}\right]}{\theta^{\prime}\left[\begin{array}{l}
1 \\
1
\end{array}\right]}=4 \pi i \frac{d}{d \tau} \log \theta^{\prime}\left[\begin{array}{l}
1 \\
1
\end{array}\right]=-\pi^{2} E_{2}(q), q=\exp (2 \pi i \tau)
$$

Proof. The proposition follows from Jacobi's triple product identity (2.5).

\section{Applications of the residue theorem}

Theorem 5.1. The sum of all the residues of an elliptic function in the fundamental parallelogram is zero. 


\subsection{Derivative formulas of level five}

Theorem 5.2. For every $\tau \in \mathbb{H}^{2}$, we have

$$
\begin{aligned}
& \frac{\theta^{\prime}\left[\begin{array}{c}
1 \\
\frac{1}{5}
\end{array}\right]}{\theta\left[\begin{array}{c}
1 \\
\frac{1}{5}
\end{array}\right]}=\theta^{\prime}\left[\begin{array}{l}
1 \\
1
\end{array}\right] \frac{\left(\theta^{5}\left[\begin{array}{c}
1 \\
\frac{1}{5}
\end{array}\right]-3 \theta^{5}\left[\begin{array}{c}
1 \\
\frac{3}{5}
\end{array}\right]\right)}{10 \theta^{3}\left[\begin{array}{c}
1 \\
\frac{1}{5}
\end{array}\right] \theta^{3}\left[\begin{array}{c}
1 \\
\frac{3}{5}
\end{array}\right]}, \\
& \frac{\theta^{\prime}\left[\begin{array}{c}
1 \\
\frac{3}{5}
\end{array}\right]}{\theta\left[\begin{array}{c}
1 \\
\frac{3}{5}
\end{array}\right]}=\theta^{\prime}\left[\begin{array}{l}
1 \\
1
\end{array}\right] \frac{\left(3 \theta^{5}\left[\begin{array}{l}
1 \\
\frac{1}{5}
\end{array}\right]+\theta^{5}\left[\begin{array}{c}
1 \\
\frac{3}{5}
\end{array}\right]\right)}{10 \theta^{3}\left[\begin{array}{l}
1 \\
\frac{1}{5}
\end{array}\right] \theta^{3}\left[\begin{array}{c}
1 \\
\frac{3}{5}
\end{array}\right]} .
\end{aligned}
$$

Proof. Consider the following elliptic functions:

$$
\varphi(z)=\frac{\theta^{3}\left[\begin{array}{l}
1 \\
1
\end{array}\right](z, \tau)}{\theta^{2}\left[\begin{array}{c}
1 \\
\frac{1}{5}
\end{array}\right](z, \tau) \theta\left[\begin{array}{c}
1 \\
\frac{3}{5}
\end{array}\right](z, \tau)} \text { and } \psi(z)=\frac{\theta^{3}\left[\begin{array}{l}
1 \\
1
\end{array}\right](z, \tau)}{\theta^{2}\left[\begin{array}{c}
1 \\
\frac{3}{5}
\end{array}\right](z, \tau) \theta\left[\begin{array}{c}
1 \\
-\frac{1}{5}
\end{array}\right](z, \tau)} .
$$

Note that, in the fundamental parallelogram, the poles of $\varphi(z)$ are $z=2 / 5$ and $z=1 / 5$. Thus, the direct calculation yields

$$
\operatorname{Res}\left(\varphi(z), \frac{2}{5}\right)=\frac{\theta^{3}\left[\begin{array}{l}
1 \\
\frac{1}{5}
\end{array}\right]}{\theta^{\prime}\left[\begin{array}{l}
1 \\
1
\end{array}\right]^{2} \theta\left[\begin{array}{l}
1 \\
\frac{3}{5}
\end{array}\right]}\left\{-3 \frac{\theta^{\prime}\left[\begin{array}{l}
1 \\
\frac{1}{5}
\end{array}\right]}{\theta\left[\begin{array}{l}
1 \\
\frac{1}{5}
\end{array}\right]}+\frac{\theta^{\prime}\left[\begin{array}{l}
1 \\
\frac{3}{5}
\end{array}\right]}{\theta\left[\begin{array}{c}
1 \\
\frac{3}{5}
\end{array}\right]}\right\}
$$

and

$$
\operatorname{Res}\left(\varphi(z), \frac{1}{5}\right)=-\frac{\theta\left[\begin{array}{l}
1 \\
\frac{3}{5}
\end{array}\right]}{\theta^{\prime}\left[\begin{array}{l}
1 \\
1
\end{array}\right]}
$$

Because $\operatorname{Res}(\varphi(z), 2 / 5)+\operatorname{Res}(\varphi(z), 1 / 5)=0$, it is understood that

$$
\frac{\theta^{\prime}\left[\begin{array}{c}
1 \\
\frac{1}{5}
\end{array}\right]}{\theta\left[\begin{array}{c}
1 \\
\frac{1}{5}
\end{array}\right]}-\frac{\theta^{\prime}\left[\begin{array}{c}
1 \\
\frac{3}{5}
\end{array}\right]}{\theta\left[\begin{array}{c}
1 \\
\frac{3}{5}
\end{array}\right]}=-\frac{\theta^{\prime}\left[\begin{array}{l}
1 \\
1
\end{array}\right] \theta^{2}\left[\begin{array}{l}
1 \\
\frac{3}{5}
\end{array}\right]}{\theta^{3}\left[\begin{array}{c}
1 \\
\frac{1}{5}
\end{array}\right]} .
$$


From $\psi(z)$, we have

$$
\frac{\theta^{\prime}\left[\begin{array}{c}
1 \\
\frac{1}{5}
\end{array}\right]}{\theta\left[\begin{array}{c}
1 \\
\frac{1}{5}
\end{array}\right]}+3 \frac{\theta^{\prime}\left[\begin{array}{c}
1 \\
\frac{3}{5}
\end{array}\right]}{\theta\left[\begin{array}{c}
1 \\
\frac{3}{5}
\end{array}\right]}=\frac{\theta^{\prime}\left[\begin{array}{l}
1 \\
1
\end{array}\right] \theta^{2}\left[\begin{array}{l}
1 \\
\frac{1}{5}
\end{array}\right]}{\theta^{3}\left[\begin{array}{c}
1 \\
\frac{3}{5}
\end{array}\right]}
$$

which proves the theorem.

Theorem 5.3. For every $\tau \in \mathbb{H}^{2}$, we have

$$
\begin{aligned}
& \frac{\theta^{\prime}\left[\begin{array}{l}
\frac{1}{5} \\
1
\end{array}\right]}{\theta\left[\begin{array}{l}
\frac{1}{5} \\
1
\end{array}\right]}=-\zeta_{5}^{3} \theta^{\prime}\left[\begin{array}{l}
1 \\
1
\end{array}\right] \frac{\left(\theta^{5}\left[\begin{array}{l}
\frac{1}{5} \\
1
\end{array}\right]+3 \theta^{5}\left[\begin{array}{l}
\frac{3}{5} \\
1
\end{array}\right]\right)}{10 \theta^{3}\left[\begin{array}{l}
\frac{1}{5} \\
1
\end{array}\right] \theta^{3}\left[\begin{array}{c}
\frac{3}{5} \\
1
\end{array}\right]}, \\
& \frac{\theta^{\prime}\left[\begin{array}{c}
\frac{3}{5} \\
1
\end{array}\right]}{\theta\left[\begin{array}{l}
\frac{3}{5} \\
1
\end{array}\right]}=-\zeta_{5}^{3} \theta^{\prime}\left[\begin{array}{l}
1 \\
1
\end{array}\right] \frac{\left(3 \theta^{5}\left[\begin{array}{l}
\frac{1}{5} \\
1
\end{array}\right]-\theta^{5}\left[\begin{array}{l}
\frac{3}{5} \\
1
\end{array}\right]\right)}{10 \theta^{3}\left[\begin{array}{l}
\frac{1}{5} \\
1
\end{array}\right] \theta^{3}\left[\begin{array}{c}
\frac{3}{5} \\
1
\end{array}\right]} .
\end{aligned}
$$

Proof. Consider the following elliptic functions:

$$
\varphi(z)=\frac{\theta^{3}\left[\begin{array}{l}
1 \\
1
\end{array}\right](z, \tau)}{\theta^{2}\left[\begin{array}{c}
\frac{1}{5} \\
1
\end{array}\right](z, \tau) \theta\left[\begin{array}{l}
\frac{3}{5} \\
1
\end{array}\right](z, \tau)} \text { and } \psi(z)=\frac{\theta^{3}\left[\begin{array}{l}
1 \\
1
\end{array}\right](z, \tau)}{\theta^{2}\left[\begin{array}{c}
\frac{3}{5} \\
1
\end{array}\right](z, \tau) \theta\left[\begin{array}{c}
-\frac{1}{5} \\
1
\end{array}\right](z, \tau)} .
$$

Then, from $\varphi(z)$ and $\psi(z)$, we have

$$
3 \frac{\theta^{\prime}\left[\begin{array}{l}
\frac{1}{5} \\
1
\end{array}\right]}{\theta\left[\begin{array}{c}
\frac{1}{5} \\
1
\end{array}\right]}-\frac{\theta^{\prime}\left[\begin{array}{c}
\frac{3}{5} \\
1
\end{array}\right]}{\theta\left[\begin{array}{c}
\frac{3}{5} \\
1
\end{array}\right]}=-\zeta_{5}^{3} \frac{\theta^{\prime}\left[\begin{array}{l}
1 \\
1
\end{array}\right] \theta^{2}\left[\begin{array}{l}
\frac{3}{5} \\
1
\end{array}\right]}{\theta^{3}\left[\begin{array}{c}
\frac{1}{5} \\
1
\end{array}\right]}
$$

and

$$
\frac{\theta^{\prime}\left[\begin{array}{c}
\frac{1}{5} \\
1
\end{array}\right]}{\theta\left[\begin{array}{c}
\frac{1}{5} \\
1
\end{array}\right]}+3 \frac{\theta^{\prime}\left[\begin{array}{c}
\frac{3}{5} \\
1
\end{array}\right]}{\theta\left[\begin{array}{c}
\frac{3}{5} \\
1
\end{array}\right]}=-\zeta_{5}^{3} \frac{\theta^{\prime}\left[\begin{array}{l}
1 \\
1
\end{array}\right] \theta^{2}\left[\begin{array}{l}
\frac{1}{5} \\
1
\end{array}\right]}{\theta^{3}\left[\begin{array}{l}
\frac{3}{5} \\
1
\end{array}\right]} .
$$

Thus, the theorem is proved. 


\subsection{Second-order theta derivatives}

Proposition 5.4. For every $\tau \in \mathbb{H}^{2}$, we have

$$
\begin{aligned}
& \frac{\theta^{\prime \prime}\left[\begin{array}{l}
1 \\
\frac{1}{5}
\end{array}\right]}{\theta\left[\begin{array}{l}
1 \\
\frac{1}{5}
\end{array}\right]}+\frac{\theta^{\prime \prime}\left[\begin{array}{l}
1 \\
\frac{3}{5}
\end{array}\right]}{\theta\left[\begin{array}{l}
1 \\
\frac{3}{5}
\end{array}\right]}-\frac{\theta^{\prime \prime \prime}\left[\begin{array}{l}
1 \\
1
\end{array}\right]}{\theta^{\prime}\left[\begin{array}{l}
1 \\
1
\end{array}\right]}+2\left\{\frac{\theta^{\prime}\left[\begin{array}{l}
1 \\
\frac{1}{5}
\end{array}\right]}{\theta\left[\begin{array}{l}
1 \\
\frac{1}{5}
\end{array}\right]}\right\}^{2}+4 \frac{\theta^{\prime}\left[\begin{array}{l}
1 \\
\frac{1}{5}
\end{array}\right]}{\theta\left[\begin{array}{l}
1 \\
\frac{1}{5}
\end{array}\right]} \cdot \frac{\theta^{\prime}\left[\begin{array}{l}
1 \\
\frac{3}{5}
\end{array}\right]}{\theta\left[\begin{array}{l}
1 \\
\frac{3}{5}
\end{array}\right]}=0, \\
& \frac{\theta^{\prime \prime}\left[\begin{array}{l}
1 \\
\frac{1}{5}
\end{array}\right]}{\theta\left[\begin{array}{l}
1 \\
\frac{1}{5}
\end{array}\right]}+2 \frac{\theta^{\prime \prime}\left[\begin{array}{c}
1 \\
\frac{3}{5}
\end{array}\right]}{\theta\left[\begin{array}{l}
1 \\
\frac{3}{5}
\end{array}\right]}-\frac{\theta^{\prime \prime \prime}\left[\begin{array}{l}
1 \\
1
\end{array}\right]}{\theta^{\prime}\left[\begin{array}{l}
1 \\
1
\end{array}\right]}-4 \frac{\theta^{\prime}\left[\begin{array}{l}
1 \\
\frac{1}{5}
\end{array}\right]}{\theta\left[\begin{array}{l}
1 \\
\frac{1}{5}
\end{array}\right]} \cdot \frac{\theta^{\prime}\left[\begin{array}{l}
1 \\
\frac{3}{5}
\end{array}\right]}{\theta\left[\begin{array}{l}
1 \\
\frac{3}{5}
\end{array}\right]}+2\left\{\frac{\theta^{\prime}\left[\begin{array}{l}
1 \\
\frac{3}{5}
\end{array}\right]}{\theta\left[\begin{array}{l}
1 \\
\frac{3}{5}
\end{array}\right]}\right\}=0 .
\end{aligned}
$$

Proof. Consider the following elliptic functions:

$$
\frac{\theta^{2}\left[\begin{array}{l}
1 \\
\frac{1}{5}
\end{array}\right](z, \tau) \theta\left[\begin{array}{l}
1 \\
\frac{3}{5}
\end{array}\right](z, \tau)}{\theta^{3}\left[\begin{array}{l}
1 \\
1
\end{array}\right](z, \tau)}, \frac{\theta\left[\begin{array}{c}
1 \\
-\frac{1}{5}
\end{array}\right](z, \tau) \theta^{2}\left[\begin{array}{l}
1 \\
\frac{3}{5}
\end{array}\right](z, \tau)}{\theta^{3}\left[\begin{array}{l}
1 \\
1
\end{array}\right](z, \tau)} .
$$

The proposition can be proved using Theorem 5.1.

Proposition 5.5. For every $\tau \in \mathbb{H}^{2}$, we have

$$
\begin{aligned}
& \frac{\theta^{\prime \prime}\left[\begin{array}{l}
\frac{1}{5} \\
1
\end{array}\right]}{\theta\left[\begin{array}{l}
\frac{1}{5} \\
1
\end{array}\right]}+\frac{\theta^{\prime \prime}\left[\begin{array}{l}
\frac{3}{5} \\
1
\end{array}\right]}{\theta\left[\begin{array}{c}
\frac{3}{5} \\
1
\end{array}\right]}-\frac{\theta^{\prime \prime \prime}\left[\begin{array}{l}
1 \\
1
\end{array}\right]}{\theta^{\prime}\left[\begin{array}{l}
1 \\
1
\end{array}\right]}+2\left\{\frac{\theta^{\prime}\left[\begin{array}{l}
\frac{1}{5} \\
1
\end{array}\right]}{\theta\left[\begin{array}{l}
\frac{1}{5} \\
1
\end{array}\right]}\right\}+4 \frac{\theta^{\prime}\left[\begin{array}{l}
\frac{1}{5} \\
1
\end{array}\right]}{\theta\left[\begin{array}{l}
\frac{1}{5} \\
1
\end{array}\right]} \cdot \frac{\theta^{\prime}\left[\begin{array}{c}
\frac{3}{5} \\
1
\end{array}\right]}{\theta\left[\begin{array}{l}
\frac{3}{5} \\
1
\end{array}\right]}=0, \\
& \frac{\theta^{\prime \prime}\left[\begin{array}{c}
\frac{1}{5} \\
1
\end{array}\right]}{\theta\left[\begin{array}{c}
\frac{1}{5} \\
1
\end{array}\right]}+2 \frac{\theta^{\prime \prime}\left[\begin{array}{c}
\frac{3}{5} \\
1
\end{array}\right]}{\theta\left[\begin{array}{l}
\frac{3}{5} \\
1
\end{array}\right]}-\frac{\theta^{\prime \prime \prime}\left[\begin{array}{l}
1 \\
1
\end{array}\right]}{\theta^{\prime}\left[\begin{array}{l}
1 \\
1
\end{array}\right]}-4 \frac{\theta^{\prime}\left[\begin{array}{c}
\frac{1}{5} \\
1
\end{array}\right]}{\theta\left[\begin{array}{c}
\frac{1}{5} \\
1
\end{array}\right]} \cdot \frac{\theta^{\prime}\left[\begin{array}{c}
\frac{3}{5} \\
1
\end{array}\right]}{\theta\left[\begin{array}{c}
\frac{3}{5} \\
1
\end{array}\right]}+2\left\{\frac{\theta^{\prime}\left[\begin{array}{c}
\frac{3}{5} \\
1
\end{array}\right]}{\theta\left[\begin{array}{c}
\frac{3}{5} \\
1
\end{array}\right]}=0 .\right.
\end{aligned}
$$

Proof. Consider the following elliptic functions:

$$
\frac{\theta^{2}\left[\begin{array}{c}
\frac{1}{5} \\
1
\end{array}\right](z, \tau) \theta\left[\begin{array}{c}
\frac{3}{5} \\
1
\end{array}\right](z, \tau)}{\theta^{3}\left[\begin{array}{l}
1 \\
1
\end{array}\right](z, \tau)}, \frac{\theta\left[\begin{array}{c}
-\frac{1}{5} \\
1
\end{array}\right](z, \tau) \theta^{2}\left[\begin{array}{l}
\frac{3}{5} \\
1
\end{array}\right](z, \tau)}{\theta^{3}\left[\begin{array}{l}
1 \\
1
\end{array}\right](z, \tau)} .
$$

The proposition can be proved using Theorem 5.1, 


\subsection{Third-order theta derivatives}

Proposition 5.6. For every $\tau \in \mathbb{H}^{2}$, we have

$$
\begin{aligned}
& \frac{\theta^{(3)}\left[\begin{array}{l}
1 \\
\frac{1}{5}
\end{array}\right]}{\theta\left[\begin{array}{l}
1 \\
\frac{1}{5}
\end{array}\right]}+3 \frac{\theta^{(3)}\left[\begin{array}{l}
1 \\
\frac{3}{5}
\end{array}\right]}{\theta\left[\begin{array}{l}
1 \\
\frac{3}{5}
\end{array}\right]}+9 \frac{\theta^{(2)}\left[\begin{array}{l}
1 \\
\frac{1}{5}
\end{array}\right]}{\theta\left[\begin{array}{l}
1 \\
\frac{1}{5}
\end{array}\right]} \frac{\theta^{(1)}\left[\begin{array}{l}
1 \\
\frac{3}{5}
\end{array}\right]}{\theta\left[\begin{array}{l}
1 \\
\frac{3}{5}
\end{array}\right]}+18 \frac{\theta^{(1)}\left[\begin{array}{l}
1 \\
\frac{1}{5}
\end{array}\right]}{\theta\left[\begin{array}{l}
1 \\
\frac{1}{5}
\end{array}\right]}\left\{\frac{\theta^{(1)}\left[\begin{array}{l}
1 \\
\frac{3}{5}
\end{array}\right]}{\theta\left[\begin{array}{l}
1 \\
\frac{3}{5}
\end{array}\right]}\right\}+9 \frac{\theta^{(1)}\left[\begin{array}{l}
1 \\
\frac{1}{5}
\end{array}\right]}{\theta\left[\begin{array}{l}
1 \\
\frac{1}{5}
\end{array}\right]} \frac{\theta^{(2)}\left[\begin{array}{l}
1 \\
\frac{3}{5}
\end{array}\right]}{\theta\left[\begin{array}{l}
1 \\
\frac{3}{5}
\end{array}\right]} \\
& +6\left\{\frac{\theta^{(1)}\left[\begin{array}{l}
1 \\
\frac{3}{5}
\end{array}\right]}{\theta\left[\begin{array}{l}
1 \\
\frac{3}{5}
\end{array}\right]}\right\}+18 \frac{\theta^{(1)}\left[\begin{array}{l}
1 \\
\frac{3}{5}
\end{array}\right]}{\theta\left[\begin{array}{l}
1 \\
\frac{3}{5}
\end{array}\right]} \frac{\theta^{(2)}\left[\begin{array}{l}
1 \\
\frac{3}{5}
\end{array}\right]}{\theta\left[\begin{array}{c}
1 \\
\frac{3}{5}
\end{array}\right]}-4 \frac{\theta^{(3)}\left[\begin{array}{l}
1 \\
1
\end{array}\right]}{\theta^{(1)}\left[\begin{array}{l}
1 \\
1
\end{array}\right]}\left(\frac{\theta^{(1)}\left[\begin{array}{l}
1 \\
\frac{1}{5}
\end{array}\right]}{\theta\left[\begin{array}{l}
1 \\
\frac{1}{5}
\end{array}\right]}+3 \frac{\theta^{(1)}\left[\begin{array}{l}
1 \\
\frac{3}{5}
\end{array}\right]}{\theta\left[\begin{array}{l}
1 \\
\frac{3}{5}
\end{array}\right]}\right]=0,
\end{aligned}
$$

and

$$
\begin{aligned}
& \frac{\theta^{(3)}\left[\begin{array}{l}
1 \\
\frac{1}{5}
\end{array}\right]}{3\left[\begin{array}{l}
1 \\
\frac{1}{5}
\end{array}\right]}-\frac{\theta^{(3)}\left[\begin{array}{l}
1 \\
\frac{3}{5}
\end{array}\right]}{\theta\left[\begin{array}{l}
1 \\
\frac{3}{5}
\end{array}\right]}+9 \frac{\theta^{(1)}\left[\begin{array}{l}
1 \\
\frac{1}{5}
\end{array}\right]}{\theta\left[\begin{array}{l}
1 \\
\frac{1}{5}
\end{array}\right]} \frac{\theta^{(2)}\left[\begin{array}{l}
1 \\
\frac{3}{5}
\end{array}\right]}{\theta\left[\begin{array}{l}
1 \\
\frac{3}{5}
\end{array}\right]}-18 \frac{\theta^{(1)}\left[\begin{array}{l}
1 \\
\frac{3}{5}
\end{array}\right]}{\theta\left[\begin{array}{l}
1 \\
\frac{3}{5}
\end{array}\right]}\left\{\frac{\theta^{(1)}\left[\begin{array}{l}
1 \\
\frac{1}{5}
\end{array}\right]}{\theta\left[\begin{array}{l}
1 \\
\frac{1}{5}
\end{array}\right]}\right\}-9 \frac{\theta^{(2)}\left[\begin{array}{l}
1 \\
\frac{1}{5}
\end{array}\right]}{\theta\left[\begin{array}{l}
1 \\
\frac{1}{5}
\end{array}\right]} \frac{\theta^{(1)}\left[\begin{array}{l}
1 \\
\frac{3}{5}
\end{array}\right]}{\theta\left[\begin{array}{l}
1 \\
\frac{3}{5}
\end{array}\right]} \\
& +6\left\{\frac{\theta^{(1)}\left[\begin{array}{l}
1 \\
\frac{1}{5}
\end{array}\right]}{\theta\left[\begin{array}{l}
1 \\
\frac{1}{5}
\end{array}\right]}\right\}+18 \frac{\theta^{(1)}\left[\begin{array}{l}
1 \\
\frac{1}{5}
\end{array}\right]}{\theta\left[\begin{array}{l}
1 \\
\frac{1}{5}
\end{array}\right]} \frac{\theta^{(2)}\left[\begin{array}{l}
1 \\
\frac{1}{5}
\end{array}\right]}{\theta\left[\begin{array}{l}
1 \\
\frac{1}{5}
\end{array}\right]}-4 \frac{\theta^{(3)}\left[\begin{array}{l}
1 \\
1
\end{array}\right]}{\theta^{(1)}\left[\begin{array}{l}
1 \\
1
\end{array}\right]}\left(3 \frac{\theta^{(1)}\left[\begin{array}{l}
1 \\
\frac{1}{5}
\end{array}\right]}{\theta\left[\begin{array}{l}
1 \\
\frac{1}{5}
\end{array}\right]}-\frac{\theta^{(1)}\left[\begin{array}{l}
1 \\
\frac{3}{5}
\end{array}\right]}{\theta\left[\begin{array}{l}
1 \\
\frac{3}{5}
\end{array}\right]}\right)=0 .
\end{aligned}
$$

Proof. Consider the following elliptic functions:

$$
\frac{\theta\left[\begin{array}{c}
1 \\
\frac{1}{5}
\end{array}\right](z) \theta^{3}\left[\begin{array}{c}
1 \\
\frac{3}{5}
\end{array}\right](z)}{\theta^{4}\left[\begin{array}{l}
1 \\
1
\end{array}\right](z)}, \frac{\theta^{3}\left[\begin{array}{c}
1 \\
\frac{1}{5}
\end{array}\right](z) \theta\left[\begin{array}{c}
1 \\
-\frac{3}{5}
\end{array}\right](z)}{\theta^{4}\left[\begin{array}{l}
1 \\
1
\end{array}\right](z)}
$$


Proposition 5.7. For every $\tau \in \mathbb{H}^{2}$, we have

$$
\begin{aligned}
& \frac{\theta^{(3)}\left[\begin{array}{c}
\frac{1}{5} \\
1
\end{array}\right]}{\theta\left[\begin{array}{c}
\frac{1}{5} \\
1
\end{array}\right]}+3 \frac{\theta^{(3)}\left[\begin{array}{c}
\frac{3}{5} \\
1
\end{array}\right]}{\theta\left[\begin{array}{c}
\frac{3}{5} \\
1
\end{array}\right]}+9 \frac{\theta^{(2)}\left[\begin{array}{c}
\frac{1}{5} \\
1
\end{array}\right]}{\theta\left[\begin{array}{c}
\frac{1}{5} \\
1
\end{array}\right]} \theta \frac{\theta^{(1)}\left[\begin{array}{c}
\frac{3}{5} \\
1
\end{array}\right]}{\theta\left[\begin{array}{c}
\frac{3}{5} \\
1
\end{array}\right]}+18 \frac{\theta^{(1)}\left[\begin{array}{c}
\frac{1}{5} \\
1
\end{array}\right]}{\theta\left[\begin{array}{c}
\frac{1}{5} \\
1
\end{array}\right]}\left\{\frac{\theta^{(1)}\left[\begin{array}{c}
\frac{3}{5} \\
1
\end{array}\right]}{\theta\left[\begin{array}{c}
\frac{3}{5} \\
1
\end{array}\right]}\right\}^{2}+9 \frac{\theta^{(1)}\left[\begin{array}{c}
\frac{1}{5} \\
1
\end{array}\right]}{\theta\left[\begin{array}{c}
\frac{1}{5} \\
1
\end{array}\right]} \frac{\theta^{(2)}\left[\begin{array}{c}
\frac{3}{5} \\
1
\end{array}\right]}{\theta\left[\begin{array}{c}
\frac{3}{5} \\
1
\end{array}\right]}
\end{aligned}
$$

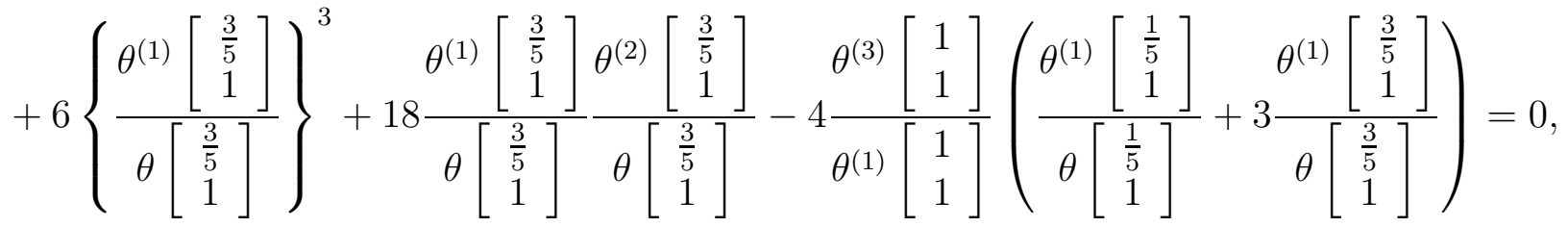

and

$$
\begin{aligned}
& 3 \frac{\theta^{(3)}\left[\begin{array}{c}
\frac{1}{5} \\
1
\end{array}\right]}{\theta\left[\begin{array}{c}
\frac{1}{5} \\
1
\end{array}\right]}-\frac{\theta^{(3)}\left[\begin{array}{c}
\frac{3}{5} \\
1
\end{array}\right]}{\theta\left[\begin{array}{c}
\frac{3}{5} \\
1
\end{array}\right]}+9 \frac{\theta^{(1)}\left[\begin{array}{c}
\frac{1}{5} \\
1
\end{array}\right]}{\theta\left[\begin{array}{c}
\frac{1}{5} \\
1
\end{array}\right]} \frac{\theta^{(2)}\left[\begin{array}{c}
\frac{3}{5} \\
1
\end{array}\right]}{\theta\left[\begin{array}{c}
\frac{3}{5} \\
1
\end{array}\right]}-18 \frac{\theta^{(1)}\left[\begin{array}{l}
\frac{3}{5} \\
1
\end{array}\right]}{\theta\left[\begin{array}{c}
\frac{3}{5} \\
1
\end{array}\right]}\left\{\frac{\theta^{(1)}\left[\begin{array}{c}
\frac{1}{5} \\
1
\end{array}\right]}{\theta\left[\begin{array}{c}
\frac{1}{5} \\
1
\end{array}\right]}\right\}-9 \frac{\theta^{(2)}\left[\begin{array}{c}
\frac{1}{5} \\
1
\end{array}\right]}{\theta\left[\begin{array}{c}
\frac{1}{5} \\
1
\end{array}\right]} \frac{\theta^{(1)}\left[\begin{array}{l}
\frac{3}{5} \\
1
\end{array}\right]}{\theta\left[\begin{array}{l}
\frac{3}{5} \\
1
\end{array}\right]}
\end{aligned}
$$

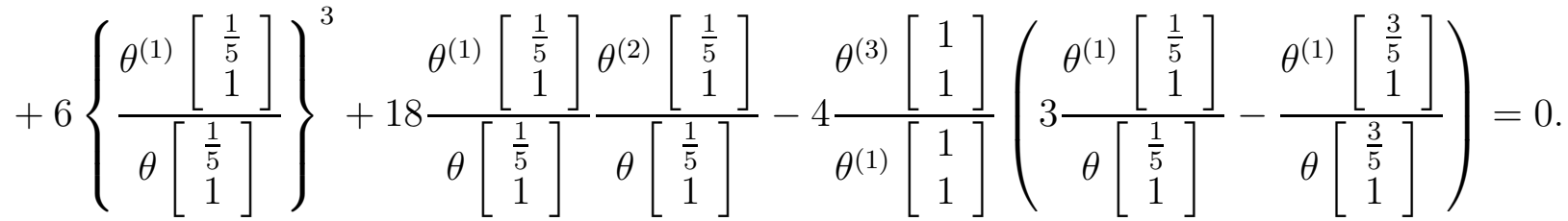

Proof. Furthermore, consider the following elliptic functions:

$$
\frac{\theta\left[\begin{array}{c}
1 \\
\frac{1}{5}
\end{array}\right](z) \theta^{3}\left[\begin{array}{c}
1 \\
\frac{3}{5}
\end{array}\right](z)}{\theta^{4}\left[\begin{array}{c}
1 \\
1
\end{array}\right](z)}, \frac{\theta^{3}\left[\begin{array}{c}
1 \\
\frac{1}{5}
\end{array}\right](z) \theta\left[\begin{array}{c}
1 \\
-\frac{3}{5}
\end{array}\right](z)}{\theta^{4}\left[\begin{array}{l}
1 \\
1
\end{array}\right](z)}
$$




\subsection{Fourth-order theta derivatives}

Proposition 5.8. For every $\tau \in \mathbb{H}^{2}$ and $j=1,3$, we have

$$
\begin{aligned}
& \frac{\theta^{(4)}\left[\begin{array}{l}
1 \\
\frac{j}{5}
\end{array}\right]}{\theta\left[\begin{array}{l}
1 \\
\frac{j}{5}
\end{array}\right]}+16 \frac{\theta^{(1)}\left[\begin{array}{l}
1 \\
\frac{j}{5}
\end{array}\right]}{\theta\left[\begin{array}{l}
1 \\
\frac{j}{5}
\end{array}\right]} \frac{\theta^{(3)}\left[\begin{array}{c}
1 \\
\frac{j}{5}
\end{array}\right]}{\theta\left[\begin{array}{c}
1 \\
\frac{j}{5}
\end{array}\right]}+12\left\{\frac{\theta^{(2)}\left[\begin{array}{l}
1 \\
\frac{j}{5}
\end{array}\right]}{\theta\left[\begin{array}{l}
1 \\
\frac{j}{5}
\end{array}\right]}\right\}^{2}+72\left\{\frac{\theta^{(1)}\left[\begin{array}{l}
1 \\
\frac{j}{5}
\end{array}\right]}{\theta\left[\begin{array}{l}
1 \\
\frac{j}{5}
\end{array}\right]}\right\}^{2} \frac{\theta^{(2)}\left[\begin{array}{l}
1 \\
\frac{j}{5}
\end{array}\right]}{\theta\left[\begin{array}{l}
1 \\
\frac{j}{5}
\end{array}\right]} \\
& +24\left\{\frac{\theta^{(1)}\left[\begin{array}{c}
1 \\
\frac{j}{5}
\end{array}\right]}{\theta\left[\begin{array}{c}
1 \\
\frac{j}{5}
\end{array}\right]}\right\}-40 \frac{\theta^{(3)}\left[\begin{array}{l}
1 \\
1
\end{array}\right]}{\theta^{(1)}\left[\begin{array}{l}
1 \\
1
\end{array}\right]}\left\{\frac{\theta^{(1)}\left[\begin{array}{l}
1 \\
\frac{j}{5}
\end{array}\right]}{\theta\left[\begin{array}{l}
1 \\
\frac{j}{5}
\end{array}\right]}\right\}^{2}-10 \frac{\theta^{(3)}\left[\begin{array}{l}
1 \\
1
\end{array}\right]}{\theta^{(1)}\left[\begin{array}{l}
1 \\
1
\end{array}\right] \theta\left[\begin{array}{l}
1 \\
\frac{j}{5}
\end{array}\right]} \\
& -\frac{1}{5} \frac{\theta^{(5)}\left[\begin{array}{l}
1 \\
1
\end{array}\right]}{\theta^{(1)}\left[\begin{array}{l}
1 \\
1
\end{array}\right]}+2\left\{\frac{\theta^{(3)}\left[\begin{array}{l}
1 \\
1
\end{array}\right]}{\theta^{(1)}\left[\begin{array}{l}
1 \\
1
\end{array}\right]}\right\}^{2}=0 \text {. }
\end{aligned}
$$

Proof. Consider the following elliptic functions: $f_{j}(z)=\theta^{5}\left[\begin{array}{l}1 \\ \frac{j}{5}\end{array}\right](z, \tau) / \theta^{5}\left[\begin{array}{l}1 \\ 1\end{array}\right](z, \tau)$.

Proposition 5.9. For every $\tau \in \mathbb{H}^{2}$ and $j=1,3$, we have

$$
\begin{aligned}
& \frac{\theta^{(4)}\left[\begin{array}{l}
\frac{j}{5} \\
1
\end{array}\right]}{\theta\left[\begin{array}{c}
\frac{j}{5} \\
1
\end{array}\right]}+16 \frac{\theta^{(1)}\left[\begin{array}{c}
\frac{j}{5} \\
1
\end{array}\right]}{\theta\left[\begin{array}{c}
\frac{j}{5} \\
1
\end{array}\right]} \frac{\theta^{(3)}\left[\begin{array}{c}
\frac{j}{5} \\
1
\end{array}\right]}{\theta\left[\begin{array}{c}
\frac{j}{5} \\
1
\end{array}\right]}+12\left\{\frac{\theta^{(2)}\left[\begin{array}{c}
\frac{j}{5} \\
1
\end{array}\right]}{\theta\left[\begin{array}{c}
\frac{j}{5} \\
1
\end{array}\right]}\right\}^{2}+72\left\{\frac{\theta^{(1)}\left[\begin{array}{c}
\frac{j}{5} \\
1
\end{array}\right]}{\theta\left[\begin{array}{c}
\frac{j}{5} \\
1
\end{array}\right]}\right\}^{2} \frac{\theta^{(2)}\left[\begin{array}{c}
\frac{j}{5} \\
1
\end{array}\right]}{\theta\left[\begin{array}{c}
\frac{j}{5} \\
1
\end{array}\right]} \\
& +24\left\{\frac{\theta^{(1)}\left[\begin{array}{c}
\frac{j}{5} \\
1
\end{array}\right]}{\theta\left[\begin{array}{c}
\frac{j}{5} \\
1
\end{array}\right]}\right\}^{4}-40 \frac{\theta^{(3)}\left[\begin{array}{l}
1 \\
1
\end{array}\right]}{\theta^{(1)}\left[\begin{array}{l}
1 \\
1
\end{array}\right]}\left\{\frac{\theta^{(1)}\left[\begin{array}{c}
\frac{j}{5} \\
1
\end{array}\right]}{\theta\left[\begin{array}{c}
\frac{j}{5} \\
1
\end{array}\right]}\right\}^{2}-10 \frac{\theta^{(3)}\left[\begin{array}{l}
1 \\
1
\end{array}\right]}{\theta^{(1)}\left[\begin{array}{l}
1 \\
1
\end{array}\right]} \frac{\theta^{(2)}\left[\begin{array}{c}
\frac{j}{5} \\
1
\end{array}\right]}{\theta\left[\begin{array}{c}
\frac{j}{5} \\
1
\end{array}\right]} \\
& -\frac{1}{5} \frac{\theta^{(5)}\left[\begin{array}{l}
1 \\
1
\end{array}\right]}{\theta^{(1)}\left[\begin{array}{l}
1 \\
1
\end{array}\right]}+2\left\{\frac{\theta^{(3)}\left[\begin{array}{l}
1 \\
1
\end{array}\right]}{\theta^{(1)}\left[\begin{array}{l}
1 \\
1
\end{array}\right]}\right\}^{2}=0
\end{aligned}
$$

Proof. Consider the following elliptic functions: $f_{j}(z)=\theta^{5}\left[\begin{array}{c}\frac{j}{5} \\ 1\end{array}\right](z, \tau) / \theta^{5}\left[\begin{array}{l}1 \\ 1\end{array}\right](z, \tau)$. 


\section{Proof of Theorem 1.1}

\subsection{Notations}

For every $\tau \in \mathbb{H}^{2}$, set $q=\exp (2 \pi i \tau)$ and

$$
X=\frac{\theta^{\prime}\left[\begin{array}{c}
1 \\
\frac{1}{5}
\end{array}\right]}{\theta\left[\begin{array}{c}
1 \\
\frac{1}{5}
\end{array}\right]}, Y=\frac{\theta^{\prime}\left[\begin{array}{c}
1 \\
\frac{3}{5}
\end{array}\right]}{\theta\left[\begin{array}{c}
1 \\
\frac{3}{5}
\end{array}\right]}, Z=\frac{\theta^{\prime \prime \prime}\left[\begin{array}{l}
1 \\
1
\end{array}\right]}{\theta^{\prime}\left[\begin{array}{l}
1 \\
1
\end{array}\right]}
$$

\subsection{ODEs for $P(q)$ and $Q(q)$}

Proof. Based on Proposition 5.4, we have

$$
\frac{\theta^{\prime \prime}\left[\begin{array}{c}
1 \\
\frac{1}{5}
\end{array}\right]}{\theta\left[\begin{array}{c}
1 \\
\frac{1}{5}
\end{array}\right]}=\frac{1}{3} Z-4 X Y-\frac{4}{3} X^{2}+\frac{2}{3} Y^{2}, \frac{\theta^{\prime \prime}\left[\begin{array}{c}
1 \\
\frac{3}{5}
\end{array}\right]}{\theta\left[\begin{array}{c}
1 \\
\frac{3}{5}
\end{array}\right]}=\frac{1}{3} Z+4 X Y+\frac{2}{3} X^{2}-\frac{4}{3} Y^{2}
$$

Based on Proposition 5.6, we obtain

$\frac{\theta^{\prime \prime \prime}\left[\begin{array}{c}1 \\ \frac{1}{5}\end{array}\right]}{\theta\left[\begin{array}{c}1 \\ \frac{1}{5}\end{array}\right]}=3 X^{3}+9 X^{2} Y-15 X Y^{2}+3 Y^{3}+X Z, \frac{\theta^{\prime \prime \prime}\left[\begin{array}{c}1 \\ \frac{3}{5}\end{array}\right]}{\theta\left[\begin{array}{c}1 \\ \frac{3}{5}\end{array}\right]}=-3 X^{3}-15 X^{2} Y-9 X Y^{2}+3 Y^{3}+Y Z$.

Because

$$
\frac{\theta^{\prime \prime \prime}\left[\begin{array}{c}
1 \\
\frac{j}{5}
\end{array}\right]}{\theta\left[\begin{array}{c}
1 \\
\frac{j}{5}
\end{array}\right]}=4 \pi i \frac{d}{d \tau}\left\{\frac{\theta^{\prime}\left[\begin{array}{l}
1 \\
\frac{j}{5}
\end{array}\right]}{\theta\left[\begin{array}{c}
1 \\
\frac{j}{5}
\end{array}\right]}\right\}+\frac{\theta^{\prime}\left[\begin{array}{l}
1 \\
\frac{j}{5}
\end{array}\right]}{\theta\left[\begin{array}{c}
1 \\
\frac{j}{5}
\end{array}\right]} \cdot \frac{\theta^{\prime \prime}\left[\begin{array}{l}
1 \\
\frac{j}{5}
\end{array}\right]}{\theta\left[\begin{array}{c}
1 \\
\frac{j}{5}
\end{array}\right]},(j=1,3),
$$

it follows that

$$
\begin{aligned}
& 4 \pi i X^{\prime}=\frac{13}{3} X^{3}+13 X^{2} Y-\frac{47}{3} X Y^{2}+3 Y^{3}+\frac{2}{3} X Z, \\
& 4 \pi i Y^{\prime}=-3 X^{3}-\frac{47}{3} X^{2} Y-13 X Y^{2}+\frac{13}{3} Y^{3}+\frac{2}{3} Y Z,
\end{aligned}
$$

where $^{\prime}=d / d \tau$. By considering $d / d \tau=2 \pi i q d / d q$, we obtain the ODEs for $P(q)$ and $Q(q)$. 


\subsection{ODE for $R(q)$}

Proof. First, note that

$$
\begin{aligned}
\frac{\theta^{(4)}\left[\begin{array}{c}
1 \\
\frac{1}{5}
\end{array}\right]}{\theta\left[\begin{array}{c}
1 \\
\frac{1}{5}
\end{array}\right]}= & 4 \pi i \frac{d}{d \tau}\left\{\frac{\theta^{\prime \prime}\left[\begin{array}{c}
1 \\
\frac{1}{5}
\end{array}\right]}{\theta\left[\begin{array}{c}
1 \\
\frac{1}{5}
\end{array}\right]}\right\}+\left\{\frac{\theta^{\prime \prime}\left[\begin{array}{c}
1 \\
\frac{1}{5}
\end{array}\right]}{\theta\left[\begin{array}{c}
1 \\
\frac{1}{5}
\end{array}\right]}\right\}^{2}, \\
= & \frac{4 \pi i}{3} \frac{d Z}{d \tau}+\frac{20}{9} X^{4}+\frac{52}{3} X^{3} Y+\frac{316}{9} X^{2} Y^{2}+\frac{44}{3} X Y^{3}-\frac{52}{9} Y^{4} \\
& -8 X Y Z-\frac{8}{3} X^{2} Z+\frac{4}{3} Y^{2} Z+\frac{1}{9} Z^{2},
\end{aligned}
$$

and

$$
\frac{\theta^{(5)}\left[\begin{array}{l}
1 \\
1
\end{array}\right]}{\theta^{(1)}\left[\begin{array}{l}
1 \\
1
\end{array}\right]}=4 \pi i \frac{d}{d \tau}\left\{\frac{\theta^{(3)}\left[\begin{array}{l}
1 \\
1
\end{array}\right]}{\theta^{(1)}\left[\begin{array}{l}
1 \\
1
\end{array}\right]}\right\}+\left\{\frac{\theta^{(3)}\left[\begin{array}{l}
1 \\
1
\end{array}\right]}{\theta^{(1)}\left[\begin{array}{l}
1 \\
1
\end{array}\right]}\right\}=4 \pi i \frac{d Z}{d \tau}+Z^{2} .
$$

By using Proposition 5.8, we have

$$
4 \pi i Z^{\prime}=\frac{10}{3} X^{4}-10 X^{3} Y-\frac{310}{3} X^{2} Y^{2}+10 X Y^{3}+\frac{10}{3} Y^{4}+\frac{2}{3} Z^{2},
$$

and

$$
\frac{\theta^{(4)}\left[\begin{array}{l}
1 \\
\frac{1}{5}
\end{array}\right]}{\theta\left[\begin{array}{l}
1 \\
\frac{1}{5}
\end{array}\right]}=\frac{10}{3} X^{4}+14 X^{3} Y+\frac{2}{3} X^{2} Y^{2}+18 X Y^{3}-\frac{14}{3} X Y^{3}+\frac{1}{3} Z^{2}-8 X Y Z-\frac{8}{3} X^{2} Z+\frac{4}{3} Y^{2} Z,
$$

where $^{\prime}=d / d \tau$. By considering $d / d \tau=2 \pi i q d / d q$, we obtain the ODE for $R(q)$.

\subsection{Note on $E_{4}$ and $E_{6}$}

Theorem 6.1. For $q \in \mathbb{C}$ with $|q|<1$, set

$$
\begin{aligned}
& P(q)=\cot \frac{2 \pi}{5}+4 \sin \frac{4 \pi}{5} \sum_{n=1}^{\infty}\left(d_{1,5}(n)-d_{4,5}(n)\right) q^{n}-4 \sin \frac{2 \pi}{5} \sum_{n=1}^{\infty}\left(d_{2,5}(n)-d_{3,5}(n)\right) q^{n} \\
& Q(q)=\cot \frac{\pi}{5}+4 \sin \frac{2 \pi}{5} \sum_{n=1}^{\infty}\left(d_{1,5}(n)-d_{4,5}(n)\right) q^{n}+4 \sin \frac{4 \pi}{5} \sum_{n=1}^{\infty}\left(d_{2,5}(n)-d_{3,5}(n)\right) q^{n}
\end{aligned}
$$


Then, we have

$$
\begin{aligned}
& E_{4}(q)=-5 P^{4}+15 P^{3} Q+155 P^{2} Q^{2}-15 P Q^{3}-5 Q^{4} \\
& E_{6}(q)=\frac{-395 P^{6}-3690 P^{5} Q-8025 P^{4} Q^{2}-8025 P^{2} Q^{4}+3690 P Q^{5}-395 Q^{6}}{8} .
\end{aligned}
$$

Proof. For the proof, we use

$$
\wp=\wp(z ; 1, \tau)=\frac{1}{3} \frac{\theta^{\prime \prime \prime}\left[\begin{array}{l}
1 \\
1
\end{array}\right]}{\theta^{\prime}\left[\begin{array}{l}
1 \\
1
\end{array}\right]}-\frac{d^{2}}{d z^{2}} \log \theta\left[\begin{array}{l}
1 \\
1
\end{array}\right](z) .
$$

Note that

$$
\wp^{\prime \prime}=6 \wp^{2}-g_{2}, g_{2}(1, \tau)=\frac{4 \pi^{4}}{3} E_{4}(q) .
$$

By substituting $z=-2 / 5$, we have

$$
g_{2}=-\frac{20}{3} X^{4}+20 X^{3} Y+\frac{620}{3} X^{2} Y^{2}-20 X Y^{3}-\frac{20}{3} Y^{4},
$$

which implies

$$
E_{4}(q)=-5 P^{4}+15 P^{3} Q+155 P^{2} Q^{2}-15 P Q^{3}-5 Q^{4} .
$$

Next, note that

$$
\left(\wp^{\prime}\right)^{2}=4 \wp^{3}-g_{2} \wp-g_{3}, g_{3}(1, \tau)=\frac{8 \pi^{6}}{27} E_{6}(q) .
$$

By substituting $z=-2 / 5$, we obtain

$$
g_{3}=-\frac{395}{27} X^{6}-\frac{410}{3} X^{5} Y-\frac{2675}{9} X^{4} Y^{2}-\frac{2675}{9} X^{2} Y^{4}+\frac{410}{3} X Y^{5}-\frac{395}{27} Y^{6}
$$

which implies

$$
E_{6}(q)=\frac{-395 P^{6}-3690 P^{5} Q-8025 P^{4} Q^{2}-8025 P^{2} Q^{4}+3690 P Q^{5}-395 Q^{6}}{8} .
$$


Corollary 6.2. For $q \in \mathbb{C}$ with $|q|<1$, set

$$
\begin{aligned}
S(q) & =\frac{\sqrt{250-110 \sqrt{5}}}{5} \prod_{n=1}^{\infty} \frac{\left(1-q^{n}\right)^{5}}{\left(1-q^{5 n}\right)^{3}}\left(1+\frac{1-\sqrt{5}}{2} q^{n}+q^{2 n}\right)^{5} \\
& =\frac{\sqrt{250-110 \sqrt{5}}}{5}\left\{1-\frac{5}{2}(1+\sqrt{5}) \sum_{n=1}^{\infty}\left(d_{1,5}(n)-d_{4,5}(n)\right) q^{n}+\frac{5}{2}(7+3 \sqrt{5}) \sum_{n=1}^{\infty}\left(d_{2,5}(n)-d_{3,5}(n)\right) q^{n}\right\}, \\
T(q) & =\frac{\sqrt{250+110 \sqrt{5}}}{5} \prod_{n=1}^{\infty} \frac{\left(1-q^{n}\right)^{5}}{\left(1-q^{5 n}\right)^{3}}\left(1+\frac{1+\sqrt{5}}{2} q^{n}+q^{2 n}\right)^{5} \\
& =\frac{\sqrt{250+110 \sqrt{5}}}{5}\left\{1+\frac{5}{2}(-1+\sqrt{5}) \sum_{n=1}^{\infty}\left(d_{1,5}(n)-d_{4,5}(n)\right) q^{n}+\frac{5}{2}(7-3 \sqrt{5}) \sum_{n=1}^{\infty}\left(d_{2,5}(n)-d_{3,5}(n)\right) q^{n}\right\} .
\end{aligned}
$$

Then we have

$$
\begin{aligned}
& E_{4}(q)=\frac{S^{4}+12 S^{3} T+14 S^{2} T^{2}-12 S T^{3}+T^{4}}{16} \\
& E_{6}(q)=-\frac{1}{64}\left(S^{2}+T^{2}\right)\left(S^{4}+18 S^{3} T+74 S^{2} T^{2}-18 S T^{3}+T^{4}\right),
\end{aligned}
$$

and

$$
\begin{aligned}
q(q: q)_{\infty}^{24} & =\frac{\left(E_{4}(q)\right)^{3}-\left(E_{6}(q)\right)^{2}}{1728}=-\frac{S^{5} T^{5}\left(S^{2}+11 S T-T^{2}\right)}{4096} \\
j(q) & =-\frac{\left(S^{4}+12 S^{3} T+14 S^{2} T^{2}-12 S T^{3}+T^{4}\right)^{3}}{S^{5} T^{5}\left(S^{2}+11 S T-T^{2}\right)}=-\frac{\left(f^{4}-12 f^{3}+14 f^{2}+12 f+1\right)^{3}}{f^{5}\left(-f^{2}+11 f+1\right)}, f=T / S .
\end{aligned}
$$

Proof. The corollary can be obtained by considering

$$
S=-3 P+Q, T=P+3 Q, \text { and } f=T / S .
$$


Corollary 6.3. For $q \in \mathbb{C}$ with $|q|<1$, set

$$
\begin{aligned}
V(q) & =\prod_{n=1}^{\infty} \frac{\left(1-q^{n}\right)^{5}}{\left(1-q^{5 n}\right)^{3}}\left(1+\frac{1-\sqrt{5}}{2} q^{n}+q^{2 n}\right)^{5} \\
& =1-\frac{5}{2}(1+\sqrt{5}) \sum_{n=1}^{\infty}\left(d_{1,5}(n)-d_{4,5}(n)\right) q^{n}+\frac{5}{2}(7+3 \sqrt{5}) \sum_{n=1}^{\infty}\left(d_{2,5}(n)-d_{3,5}(n)\right) q^{n}, \\
W(q) & =\prod_{n=1}^{\infty} \frac{\left(1-q^{n}\right)^{5}}{\left(1-q^{5 n}\right)^{3}}\left(1+\frac{1+\sqrt{5}}{2} q^{n}+q^{2 n}\right)^{5} \\
& =1+\frac{5}{2}(-1+\sqrt{5}) \sum_{n=1}^{\infty}\left(d_{1,5}(n)-d_{4,5}(n)\right) q^{n}+\frac{5}{2}(7-3 \sqrt{5}) \sum_{n=1}^{\infty}\left(d_{2,5}(n)-d_{3,5}(n)\right) q^{n} .
\end{aligned}
$$

Then we have

$$
\begin{aligned}
E_{4}(q)= & \frac{(123-55 \sqrt{5}) V^{4}+12(-11+5 \sqrt{5}) V^{3} W+28 V^{2} W^{2}-12(11+5 \sqrt{5}) V W^{3}+(123+55 \sqrt{5}) W^{4}}{10}, \\
E_{6}(q)= & \frac{1}{25}(-1525+682 \sqrt{5}) V^{6}-\frac{9}{25}(-275+123 \sqrt{5}) V^{5} W+\frac{3}{2}(-25+11 \sqrt{5}) V^{4} W^{2} \\
& -\frac{3}{2}(25+11 \sqrt{5}) V^{2} W^{4}+\frac{9}{25}(275+123 \sqrt{5}) V W^{5}-\frac{1}{25}(1525+682 \sqrt{5}) W^{6},
\end{aligned}
$$

and

$$
\begin{aligned}
q(q ; q)_{\infty}^{24} & =-\frac{1}{250} V^{5} W^{5}(V-W)((-11+5 \sqrt{5}) V+(11+5 \sqrt{5}) W) \\
& =\frac{1}{250}(11-5 \sqrt{5}) V^{7} W^{5}-\frac{11}{125} V^{6} W^{6}+\frac{1}{250}(11+5 \sqrt{5}) V^{5} W^{7}
\end{aligned}
$$

Theorem 6.4. For every $\tau \in \mathbb{H}^{2}$, we have

$$
G_{4}(\tau)=\frac{\theta^{\prime}\left[\begin{array}{c}
1 \\
1
\end{array}\right]^{4}}{720 \cdot \theta^{12}\left[\begin{array}{c}
1 \\
\frac{1}{5}
\end{array}\right] \theta^{12}\left[\begin{array}{c}
1 \\
\frac{3}{5}
\end{array}\right]}\left(A^{4}-12 A^{3} B+14 A^{2} B^{2}+12 A B^{3}+B^{4}\right),
$$

and

$$
G_{6}(\tau)=\frac{-\theta^{\prime}\left[\begin{array}{c}
1 \\
1
\end{array}\right]^{6}}{30240 \cdot \theta^{18}\left[\begin{array}{c}
1 \\
\frac{1}{5}
\end{array}\right] \theta^{18}\left[\begin{array}{c}
1 \\
\frac{3}{5}
\end{array}\right]}\left(A^{2}+B^{2}\right)\left(A^{4}-18 A^{3} B+74 A^{2} B^{2}+18 A B^{3}+B^{4}\right),
$$


where

$$
A=\theta^{5}\left[\begin{array}{c}
1 \\
\frac{1}{5}
\end{array}\right], B=\theta^{5}\left[\begin{array}{c}
1 \\
\frac{3}{5}
\end{array}\right] .
$$

Proof. The theorem is based on equations (6.3) and (6.4) and the derivative formulas of Theorem 5.2.

Theorem 6.5. For every $\tau \in \mathbb{H}^{2}$, we have

$$
\Delta=g_{2}^{3}-27 g_{3}^{2}=\frac{\theta^{\prime}\left[\begin{array}{l}
1 \\
1
\end{array}\right]^{12}}{\theta^{11}\left[\begin{array}{c}
1 \\
\frac{1}{5}
\end{array}\right] \theta^{11}\left[\begin{array}{c}
1 \\
\frac{3}{5}
\end{array}\right]}\left(\theta^{10}\left[\begin{array}{c}
1 \\
\frac{1}{5}
\end{array}\right]-11 \theta^{5}\left[\begin{array}{c}
1 \\
\frac{1}{5}
\end{array}\right] \theta^{5}\left[\begin{array}{c}
1 \\
\frac{3}{5}
\end{array}\right]-\theta^{10}\left[\begin{array}{c}
1 \\
\frac{3}{5}
\end{array}\right]\right),
$$

and

$$
j=\frac{12^{3} g_{2}^{3}}{\Delta}=\frac{\left(f^{4}(\tau)-12 f^{3}(\tau)+14 f^{2}(\tau)+12 f(\tau)+1\right)^{3}}{f^{5}(\tau)\left(f^{2}(\tau)-11 f(\tau)-1\right)}
$$

where

$$
f(\tau)=\theta^{5}\left[\begin{array}{c}
1 \\
\frac{1}{5}
\end{array}\right] / \theta^{5}\left[\begin{array}{c}
1 \\
\frac{3}{5}
\end{array}\right]=\frac{11+5 \sqrt{5}}{2} \prod_{n=1}^{\infty} \frac{\left(1+\frac{1+\sqrt{5}}{2} q^{n}+q^{2 n}\right)^{5}}{\left(1+\frac{1-\sqrt{5}}{2} q^{n}+q^{2 n}\right)^{5}}, q=\exp (2 \pi i \tau) .
$$

Proof. By using equations (6.3) and (6.4), we have

$$
\begin{aligned}
& \Delta=-25(3 X-Y)^{5}(X+3 Y)^{5}\left(X^{2}+4 X Y-Y^{2}\right)=\frac{\theta^{\prime}\left[\begin{array}{l}
1 \\
1
\end{array}\right]^{12}}{\theta^{36}\left[\begin{array}{c}
1 \\
\frac{1}{5}
\end{array}\right] \theta^{36}\left[\begin{array}{l}
1 \\
\frac{3}{5}
\end{array}\right]} A^{5} B^{5}\left(A^{2}-11 A B-B^{2}\right), \\
& j=\frac{320\left(X^{4}-3 X^{3} Y-31 X^{2} Y^{2}+3 X Y^{3}+Y^{4}\right)^{3}}{27(3 X-Y)^{5}(X+3 Y)^{5}\left(X^{2}+4 X Y-Y^{2}\right)}=\frac{\left(A^{4}-12 A^{3} B+14 A^{2} B^{2}+12 A B^{3}+B^{4}\right)^{3}}{A^{5} B^{5}\left(A^{2}-11 A B-B^{2}\right)},
\end{aligned}
$$

where

$$
A=\theta^{5}\left[\begin{array}{c}
1 \\
\frac{1}{5}
\end{array}\right], B=\theta^{5}\left[\begin{array}{c}
1 \\
\frac{3}{5}
\end{array}\right]
$$

Corollary 6.6. For $q \in \mathbb{C}$ with $|q|<1$, set

$$
\begin{aligned}
& P(q)=\cot \frac{2 \pi}{5}+4 \sin \frac{4 \pi}{5} \sum_{n=1}^{\infty}\left(d_{1,5}(n)-d_{4,5}(n)\right) q^{n}-4 \sin \frac{2 \pi}{5} \sum_{n=1}^{\infty}\left(d_{2,5}(n)-d_{3,5}(n)\right) q^{n} \\
& Q(q)=\cot \frac{\pi}{5}+4 \sin \frac{2 \pi}{5} \sum_{n=1}^{\infty}\left(d_{1,5}(n)-d_{4,5}(n)\right) q^{n}+4 \sin \frac{4 \pi}{5} \sum_{n=1}^{\infty}\left(d_{2,5}(n)-d_{3,5}(n)\right) q^{n}
\end{aligned}
$$


Then, we have

$$
j(\tau)=j(q)=\frac{320\left(P^{4}-3 P^{3} Q-31 P^{2} Q^{2}+3 P Q^{3}+Q^{4}\right)^{3}}{27(3 P-Q)^{5}(P+3 Q)^{5}\left(P^{2}+4 P Q-Q^{2}\right)},
$$

and

$$
f(\tau)=\theta^{5}\left[\begin{array}{c}
1 \\
\frac{1}{5}
\end{array}\right] / \theta^{5}\left[\begin{array}{c}
1 \\
\frac{3}{5}
\end{array}\right]=-\frac{P+3 Q}{3 P-Q}, q=\exp (2 \pi i \tau) .
$$

Proof. The expression of $f(\tau)$ follows from the derivative formulas (5.1) and (5.2), which implies that

$$
f(\tau)=-\frac{X+3 Y}{3 X-Y}
$$

Theorem 6.7. For every $\tau \in \mathbb{H}^{2}$, we have

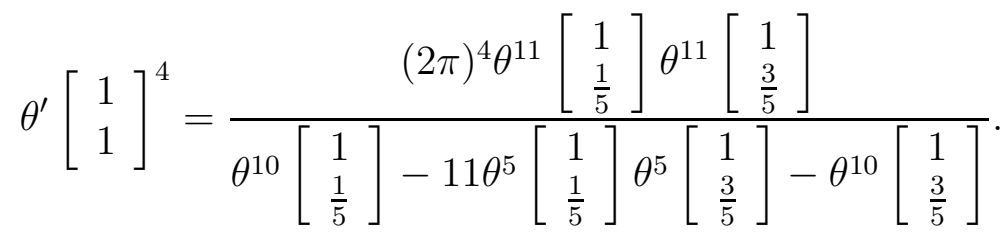

Proof. Recall the following formula from Apostol [1, pp. 51]:

$$
\Delta=(2 \pi)^{12} \eta^{24}(\tau)=(2 \pi)^{4} \theta^{\prime}\left[\begin{array}{l}
1 \\
1
\end{array}\right]^{8} .
$$

The theorem follows from Theorem 6.5.

\section{Theorem 6.8.}

(1) For each positive integer $k$, the Eisenstein series $G_{4 k}(\tau)$ can be expressed using a rational expression as follows:

$$
\theta\left[\begin{array}{c}
1 \\
\frac{1}{5}
\end{array}\right](0, \tau) \text {, and } \theta\left[\begin{array}{c}
1 \\
\frac{3}{5}
\end{array}\right](0, \tau)
$$

(2) For each positive integer $k$, the Eisenstein series $G_{4 k+2}(\tau)$ can be expressed using a rational expression as follows:

$$
\theta\left[\begin{array}{c}
1 \\
\frac{1}{5}
\end{array}\right](0, \tau), \theta\left[\begin{array}{c}
1 \\
\frac{3}{5}
\end{array}\right](0, \tau) \text {, and } \theta^{\prime}\left[\begin{array}{l}
1 \\
1
\end{array}\right](0, \tau) .
$$


Proof. From the study by Apostol [1, pp. 118], we recall that for $k=2,3,4 \ldots$,

$$
G_{2 k}(\tau)=\sum_{a, b} c_{a, b} G_{4}^{4}(\tau) G_{6}^{b}(\tau)
$$

where $c_{a, b}$ are complex numbers, and the sum is extended over all integers $a \geq 0, b \geq 0$ such that $4 a+6 b=k$. The theorem follows from Theorems 6.4 and 6.7 .

\subsection{Proof of Ramanujan's ODEs (1.3)}

Theorem 6.9. For $q \in \mathbb{C}$ with $|q|<1$, we have

$$
q \frac{d E_{2}}{d q}=\frac{\left(E_{2}\right)^{2}-E_{4}}{12}, q \frac{d E_{4}}{d q}=\frac{E_{2} E_{4}-E_{6}}{3}, q \frac{d E_{6}}{d q}=\frac{E_{2} E_{6}-\left(E_{4}\right)^{2}}{2} .
$$

Proof. According to Theorems 1.1 and 6.1.

$$
q \frac{d}{d q} E_{2}=q \frac{d}{d q} R=\frac{5 P^{4}-15 P^{3} Q-155 P^{2} Q^{2}+15 P Q^{3}+5 Q^{4}+R^{2}}{12}=\frac{E_{2}^{2}-E_{4}}{12} .
$$

Next, it is observed that

$$
\begin{aligned}
q \frac{d}{d q} E_{4}= & q \frac{d}{d q}\left(-5 P^{4}+15 P^{3} Q+155 P^{2} Q^{2}-15 P Q^{3}-5 Q^{4}\right) \\
= & \frac{395 P^{6}}{24}+\frac{615 P^{5} Q}{4}+\frac{2675 P^{4} Q^{2}}{8}+\frac{2675 P^{2} Q^{4}}{8}-\frac{615 P Q^{5}}{4}+\frac{395 Q^{6}}{24} \\
& -\frac{5 P^{4} R}{3}+5 P^{3} Q R+\frac{155}{3} P^{2} Q^{2} R-5 P Q^{3} R-\frac{5 Q^{4} R}{3} \\
= & \frac{E_{2} E_{4}-E_{6}}{3} .
\end{aligned}
$$

Finally, we obtain

$$
\begin{aligned}
& q \frac{d}{d q} E_{6}=q \frac{d}{d q}\left\{\frac{-395 P^{6}-3690 P^{5} Q-8025 P^{4} Q^{2}-8025 P^{2} Q^{4}+3690 P Q^{5}-395 Q^{6}}{8}\right\} \\
= & -\frac{25 P^{8}}{2}+75 P^{7} Q+\frac{1325 P^{6} Q^{2}}{2}-2400 P^{5} Q^{3}-\frac{23625 P^{4} Q^{4}}{2} \\
& +2400 P^{3} Q^{5}+\frac{1325 P^{2} Q^{6}}{2}-75 P Q^{7}-\frac{25 Q^{8}}{2} \\
& -\frac{395 P^{6} R}{16}-\frac{1845}{8} P^{5} Q R-\frac{8025}{16} P^{4} Q^{2} R-\frac{8025}{16} P^{2} Q^{4} R+\frac{1845}{8} P Q^{5} R-\frac{395 Q^{6} R}{16} \\
= & \frac{E_{2} E_{6}-\left(E_{4}\right)^{2}}{2} .
\end{aligned}
$$




\section{Proof of Theorem 1.2}

\subsection{Notations}

For every $\tau \in \mathbb{H}^{2}$, set $y=\exp (2 \pi i \tau / 5)$ and

$$
X=\frac{\theta^{\prime}\left[\begin{array}{c}
\frac{1}{5} \\
1
\end{array}\right]}{\theta\left[\begin{array}{c}
\frac{1}{5} \\
1
\end{array}\right]}, Y=\frac{\theta^{\prime}\left[\begin{array}{l}
\frac{3}{5} \\
1
\end{array}\right]}{\theta\left[\begin{array}{c}
\frac{3}{5} \\
1
\end{array}\right]}, Z=\frac{\theta^{\prime \prime \prime}\left[\begin{array}{l}
1 \\
1
\end{array}\right]}{\theta^{\prime}\left[\begin{array}{l}
1 \\
1
\end{array}\right]}
$$

\subsection{ODEs for $P(q)$ and $Q(q)$}

Proof. According to Proposition 5.5, we have

$$
\frac{\theta^{\prime \prime}\left[\begin{array}{l}
\frac{1}{5} \\
1
\end{array}\right]}{\theta\left[\begin{array}{c}
\frac{1}{5} \\
1
\end{array}\right]}=\frac{1}{3} Z-4 X Y-\frac{4}{3} X^{2}+\frac{2}{3} Y^{2}, \frac{\theta^{\prime \prime}\left[\begin{array}{c}
\frac{3}{5} \\
1
\end{array}\right]}{\theta\left[\begin{array}{c}
\frac{3}{5} \\
1
\end{array}\right]}=\frac{1}{3} Z+4 X Y+\frac{2}{3} X^{2}-\frac{4}{3} Y^{2}
$$

According to Proposition 5.7, we obtain

$\frac{\theta^{\prime \prime \prime}\left[\begin{array}{l}\frac{1}{5} \\ 1\end{array}\right]}{\theta\left[\begin{array}{c}\frac{1}{5} \\ 1\end{array}\right]}=3 X^{3}+9 X^{2} Y-15 X Y^{2}+3 Y^{3}+X Z, \frac{\theta^{\prime \prime \prime}\left[\begin{array}{c}\frac{3}{5} \\ 1\end{array}\right]}{\theta\left[\begin{array}{c}\frac{3}{5} \\ 1\end{array}\right]}=-3 X^{3}-15 X^{2} Y-9 X Y^{2}+3 Y^{3}+Y Z$.

Because

$$
\frac{\theta^{\prime \prime \prime}\left[\begin{array}{c}
\frac{j}{5} \\
1
\end{array}\right]}{\theta\left[\begin{array}{c}
\frac{j}{5} \\
1
\end{array}\right]}=4 \pi i \frac{d}{d \tau}\left\{\frac{\theta^{\prime}\left[\begin{array}{c}
\frac{j}{5} \\
1
\end{array}\right]}{\theta\left[\begin{array}{c}
\frac{j}{5} \\
1
\end{array}\right]}\right\}+\frac{\theta^{\prime}\left[\begin{array}{c}
\frac{j}{5} \\
1
\end{array}\right]}{\theta\left[\begin{array}{c}
\frac{j}{5} \\
1
\end{array}\right]} \cdot \frac{\theta^{\prime \prime}\left[\begin{array}{c}
\frac{j}{5} \\
1
\end{array}\right]}{\theta\left[\begin{array}{c}
\frac{j}{5} \\
1
\end{array}\right]},(j=1,3),
$$

we get

$$
\begin{aligned}
& 4 \pi i X^{\prime}=\frac{13}{3} X^{3}+13 X^{2} Y-\frac{47}{3} X Y^{2}+3 Y^{3}+\frac{2}{3} X Z, \\
& 4 \pi i Y^{\prime}=-3 X^{3}-\frac{47}{3} X^{2} Y-13 X Y^{2}+\frac{13}{3} Y^{3}+\frac{2}{3} Y Z,
\end{aligned}
$$

where $^{\prime}=d / d \tau$. Thus, by changing $\tau \rightarrow 5 \tau$, we obtain the ODEs for $P(q)$ and $Q(q)$. 


\subsection{ODE for $R(q)$}

Proof. First, note that

$$
\begin{aligned}
\frac{\theta^{(4)}\left[\begin{array}{l}
\frac{1}{5} \\
1
\end{array}\right]}{\theta\left[\begin{array}{c}
\frac{1}{5} \\
1
\end{array}\right]}= & 4 \pi i \frac{d}{d \tau}\left\{\frac{\theta^{\prime \prime}\left[\begin{array}{l}
\frac{1}{5} \\
1
\end{array}\right]}{\theta\left[\begin{array}{c}
\frac{1}{5} \\
1
\end{array}\right]}\right\}+\left\{\frac{\theta^{\prime \prime}\left[\begin{array}{c}
\frac{1}{5} \\
1
\end{array}\right]}{\theta\left[\begin{array}{c}
\frac{1}{5} \\
1
\end{array}\right]}\right\}^{2}, \\
= & \frac{4 \pi i}{3} \frac{d Z}{d \tau}+\frac{20}{9} X^{4}+\frac{52}{3} X^{3} Y+\frac{316}{9} X^{2} Y^{2}+\frac{44}{3} X Y^{3}-\frac{52}{9} Y^{4} \\
& -8 X Y Z-\frac{8}{3} X^{2} Z+\frac{4}{3} Y^{2} Z+\frac{1}{9} Z^{2},
\end{aligned}
$$

and

$$
\frac{\theta^{(5)}\left[\begin{array}{l}
1 \\
1
\end{array}\right]}{\theta^{(1)}\left[\begin{array}{l}
1 \\
1
\end{array}\right]}=4 \pi i \frac{d}{d \tau}\left\{\frac{\theta^{(3)}\left[\begin{array}{l}
1 \\
1
\end{array}\right]}{\theta^{(1)}\left[\begin{array}{l}
1 \\
1
\end{array}\right]}\right\}+\left\{\frac{\theta^{(3)}\left[\begin{array}{l}
1 \\
1
\end{array}\right]}{\theta^{(1)}\left[\begin{array}{l}
1 \\
1
\end{array}\right]}\right\}=4 \pi i \frac{d Z}{d \tau}+Z^{2}
$$

Thus, according to Proposition 5.9, we have

$$
4 \pi i Z^{\prime}=\frac{10}{3} X^{4}-10 X^{3} Y-\frac{310}{3} X^{2} Y^{2}+10 X Y^{3}+\frac{10}{3} Y^{4}+\frac{2}{3} Z^{2},
$$

and

$$
\frac{\theta^{(4)}\left[\begin{array}{l}
\frac{1}{5} \\
1
\end{array}\right]}{\theta\left[\begin{array}{c}
\frac{1}{5} \\
1
\end{array}\right]}=\frac{10}{3} X^{4}+14 X^{3} Y+\frac{2}{3} X^{2} Y^{2}+18 X Y^{3}-\frac{14}{3} X Y^{3}+\frac{1}{3} Z^{2}-8 X Y Z-\frac{8}{3} X^{2} Z+\frac{4}{3} Y^{2} Z,
$$

where $^{\prime}=d / d \tau$. Thus, by changing $\tau \rightarrow 5 \tau$, we obtain the ODE for $R(q)$.

Theorem 7.1. For $q \in \mathbb{C}$ with $|q|<1$, set

$$
P(q)=1+10 \sum_{n=1}^{\infty}\left(d_{2,5}(n)-d_{3,5}(n)\right) q^{n}, Q(q)=3+10 \sum_{n=1}^{\infty}\left(d_{1,5}(n)-d_{4,5}(n)\right) q^{n} .
$$

Then, we have

$$
\begin{aligned}
& E_{4}\left(q^{5}\right)=\frac{-P^{4}+3 P^{3} Q+31 P^{2} Q^{2}-3 P Q^{3}-Q^{4}}{125}, \\
& E_{6}\left(q^{5}\right)=\frac{79 P^{6}+738 P^{5} Q+1605 P^{4} Q^{2}+1605 P^{2} Q^{4}-738 P Q^{5}+79 Q^{6}}{24} .
\end{aligned}
$$


Proof. For the proof, we use

$$
\wp=\wp(z ; 1, \tau)=\frac{1}{3} \frac{\theta^{\prime \prime \prime}\left[\begin{array}{l}
1 \\
1
\end{array}\right]}{\theta^{\prime}\left[\begin{array}{l}
1 \\
1
\end{array}\right]}-\frac{d^{2}}{d z^{2}} \log \theta\left[\begin{array}{l}
1 \\
1
\end{array}\right](z) .
$$

First, note that

$$
\wp^{\prime \prime}=6 \wp^{2}-g_{2}, g_{2}(1, \tau)=\frac{4 \pi^{4}}{3} E_{4}(q) .
$$

Then, by substituting $z=-2 \tau / 5$, we have

$$
g_{2}=-\frac{20}{3} X^{4}+20 X^{3} Y+\frac{620}{3} X^{2} Y^{2}-20 X Y^{3}-\frac{20}{3} Y^{4},
$$

which implies

$$
E_{4}\left(q^{5}\right)=\frac{-P^{4}+3 P^{3} Q+31 P^{2} Q^{2}-3 P Q^{3}-Q^{4}}{125} .
$$

Next, it is observed that

$$
\left(\wp^{\prime}\right)^{2}=4 \wp^{3}-g_{2} \wp-g_{3}, g_{3}(1, \tau)=\frac{8 \pi^{6}}{27} E_{6}(q) .
$$

Thus, by substituting $z=-2 \tau / 5$, we obtain

$$
g_{3}=-\frac{395}{27} X^{6}-\frac{410}{3} X^{5} Y-\frac{2675}{9} X^{4} Y^{2}-\frac{2675}{9} X^{2} Y^{4}+\frac{410}{3} X Y^{5}-\frac{395}{27} Y^{6},
$$

which implies

$$
E_{6}\left(q^{5}\right)=\frac{79 P^{6}+738 P^{5} Q+1605 P^{4} Q^{2}+1605 P^{2} Q^{4}-738 P Q^{5}+79 Q^{6}}{25000} .
$$

Corollary 7.2. For $q \in \mathbb{C}$ with $|q|<1$, set

$S(q)=q \prod_{n=1}^{\infty} \frac{\left(1-q^{n}\right)^{2}}{\left(1-q^{5 n-2}\right)^{5}\left(1-q^{5 n-3}\right)^{5}}=\sum_{n=1}^{\infty}\left(d_{1,5}(n)-d_{4,5}(n)\right) q^{n}-3 \sum_{n=1}^{\infty}\left(d_{2,5}(n)-d_{3,5}(n)\right) q^{n}$,

and

$T(q)=\prod_{n=1}^{\infty} \frac{\left(1-q^{n}\right)^{2}}{\left(1-q^{5 n-1}\right)^{5}\left(1-q^{5 n-4}\right)^{5}}=1+3 \sum_{n=1}^{\infty}\left(d_{1,5}(n)-d_{4,5}(n)\right) q^{n}+\sum_{n=1}^{\infty}\left(d_{2,5}(n)-d_{3,5}(n)\right) q^{n}$. 
Then, we have

$$
\begin{aligned}
& E_{4}\left(q^{5}\right)=S^{4}+12 S^{3} T+14 S^{2} T^{2}-12 S T^{3}+T^{4}, \\
& E_{6}\left(q^{5}\right)=\left(S^{2}+T^{2}\right)\left(S^{4}+18 S^{3} T+74 S^{2} T^{2}-18 S T^{3}+T^{4}\right),
\end{aligned}
$$

and

$$
\begin{aligned}
q^{5}\left(q^{5} ; q^{5}\right)_{\infty} & =-S^{5} T^{5}\left(S^{2}+11 S T-T^{2}\right) \\
j\left(q^{5}\right) & =-\frac{\left(S^{4}+12 S^{3} T+14 S^{2} T^{2}-12 S T^{3}+T^{4}\right)^{3}}{S^{5} T^{5}\left(S^{2}+11 S T-T^{2}\right)}=-\frac{\left(g^{4}+12 g^{3}+14 g^{2}-12 g+1\right)^{3}}{g^{5}\left(g^{2}+11 g-1\right)}, g=S / T .
\end{aligned}
$$

Proof. The corollary can be obtained by considering

$$
S=-\frac{3}{10} P+\frac{1}{10} Q, T=\frac{1}{10} P+\frac{3}{10} Q .
$$

Theorem 7.3. For every $\tau \in \mathbb{H}^{2}$, we have

$$
G_{4}(\tau)=\frac{\zeta_{5}^{2} \theta^{\prime}\left[\begin{array}{l}
1 \\
1
\end{array}\right]^{4}}{720 \cdot \theta^{12}\left[\begin{array}{l}
\frac{1}{5} \\
1
\end{array}\right] \theta^{12}\left[\begin{array}{l}
\frac{3}{5} \\
1
\end{array}\right]}\left(A^{4}+12 A^{3} B+14 A^{2} B^{2}-12 A B^{3}+B^{4}\right),
$$

and

$$
G_{6}(\tau)=\frac{-\zeta_{5}^{3} \theta^{\prime}\left[\begin{array}{l}
1 \\
1
\end{array}\right]^{6}}{30240 \cdot \theta^{18}\left[\begin{array}{l}
\frac{1}{5} \\
1
\end{array}\right] \theta^{18}\left[\begin{array}{l}
\frac{3}{5} \\
1
\end{array}\right]}\left(A^{2}+B^{2}\right)\left(A^{4}+18 A^{3} B+74 A^{2} B^{2}-18 A B^{3}+B^{4}\right),
$$

where $\zeta_{5}=\exp (2 \pi i / 5)$, and

$$
A=\theta^{5}\left[\begin{array}{c}
\frac{1}{5} \\
1
\end{array}\right], B=\theta^{5}\left[\begin{array}{c}
\frac{3}{5} \\
1
\end{array}\right] .
$$

Proof. The theorem follows from equations (7.3) and (7.4) and the derivative formulas of Theorem 5.3 . 
Theorem 7.4. For every $\tau \in \mathbb{H}^{2}$, we have

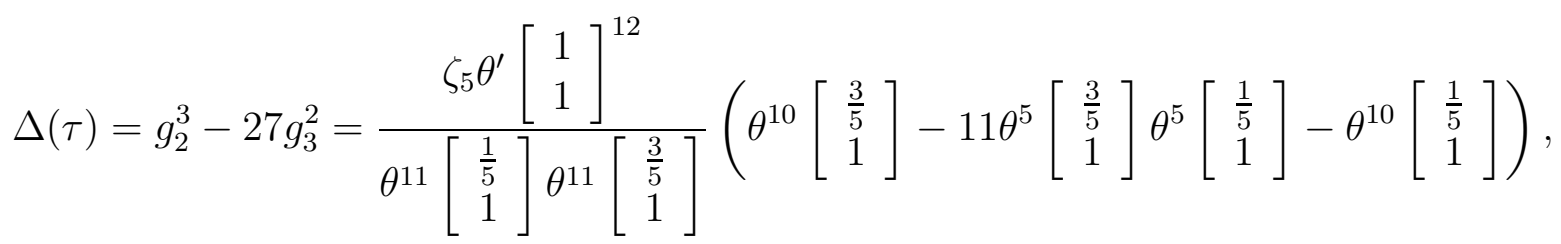

and

$$
j(5 \tau)=\frac{12^{3} g_{2}(5 \tau)^{3}}{\Delta(5 \tau)}=\frac{\left(g^{4}(\tau)-12 g^{3}(\tau)+14 g^{2}(\tau)+12 g(\tau)+1\right)^{3}}{g^{5}(\tau)\left(g^{2}(\tau)-11 g(\tau)-1\right)},
$$

where $\zeta_{5}=\exp (2 \pi i / 5)$,

$$
g(\tau)=\theta^{5}\left[\begin{array}{c}
\frac{3}{5} \\
1
\end{array}\right](0,5 \tau) / \theta^{5}\left[\begin{array}{c}
\frac{1}{5} \\
1
\end{array}\right](0,5 \tau)=-q \prod_{n=1}^{\infty} \frac{\left(1-q^{5 n-1}\right)^{5}\left(1-q^{5 n-4}\right)^{5}}{\left(1-q^{5 n-2}\right)^{5}\left(1-q^{5 n-3}\right)^{5}}, q=\exp (2 \pi i \tau) .
$$

Proof. Based on equations (17.3) and (7.4), we have

$$
\begin{gathered}
\Delta=-25(3 X-Y)^{5}(X+3 Y)^{5}\left(X^{2}+4 X Y-Y^{2}\right)=\frac{-\zeta_{5} \theta^{\prime}\left[\begin{array}{l}
1 \\
1
\end{array}\right]^{12}}{\theta^{36}\left[\begin{array}{l}
\frac{1}{5} \\
1
\end{array}\right] \theta^{36}\left[\begin{array}{l}
\frac{3}{5} \\
1
\end{array}\right]} A^{5} B^{5}\left(A^{2}+11 A B-B^{2}\right), \\
j=\frac{320\left(X^{4}-3 X^{3} Y-31 X^{2} Y^{2}+3 X Y^{3}+Y^{4}\right)^{3}}{27(3 X-Y)^{5}(X+3 Y)^{5}\left(X^{2}+4 X Y-Y^{2}\right)}=-\frac{\left(A^{4}+12 A^{3} B+14 A^{2} B^{2}-12 A B^{3}+B^{4}\right)^{3}}{A^{5} B^{5}\left(A^{2}+11 A B-B^{2}\right)},
\end{gathered}
$$

where

$$
A=\theta^{5}\left[\begin{array}{c}
\frac{1}{5} \\
1
\end{array}\right], B=\theta^{5}\left[\begin{array}{c}
\frac{3}{5} \\
1
\end{array}\right]
$$

Corollary 7.5. For $q \in \mathbb{C}$ with $|q|<1$, set

$$
P(q)=1+10 \sum_{n=1}^{\infty}\left(d_{2,5}(n)-d_{3,5}(n)\right) q^{n}, Q(q)=3+10 \sum_{n=1}^{\infty}\left(d_{1,5}(n)-d_{4,5}(n)\right) q^{n} .
$$

Then, we have

$$
j(5 \tau)=j\left(q^{5}\right)=\frac{320\left(P^{4}-3 P^{3} Q-31 P^{2} Q^{2}+3 P Q^{3}+Q^{4}\right)^{3}}{27(3 P-Q)^{5}(P+3 Q)^{5}\left(P^{2}+4 P Q-Q^{2}\right)},
$$

and

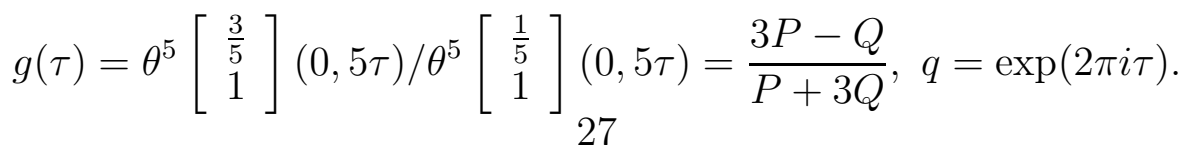


Proof. The expression of $g(\tau)$ follows from the derivative formulas (5.3) and (5.4), which imply that

$$
g(\tau / 5)=\frac{3 X-Y}{X+3 Y}
$$

Theorem 7.6. For every $\tau \in \mathbb{H}^{2}$, set

$$
f(\tau)=\frac{\theta^{5}\left[\begin{array}{c}
1 \\
\frac{1}{5}
\end{array}\right](0, \tau)}{\theta^{5}\left[\begin{array}{c}
1 \\
\frac{3}{5}
\end{array}\right](0, \tau)}=\frac{11+5 \sqrt{5}}{2} \prod_{n=1}^{\infty} \frac{\left(1+\frac{1+\sqrt{5}}{2} q^{n}+q^{2 n}\right)^{5}}{\left(1+\frac{1-\sqrt{5}}{2} q^{n}+q^{2 n}\right)^{5}}
$$

and

$$
g(\tau)=\frac{\theta^{5}\left[\begin{array}{c}
\frac{3}{5} \\
1
\end{array}\right](0,5 \tau)}{\theta^{5}\left[\begin{array}{c}
\frac{1}{5} \\
1
\end{array}\right](0,5 \tau)}=-q \prod_{n=1}^{\infty} \frac{\left(1-q^{5 n-1}\right)^{5}\left(1-q^{5 n-4}\right)^{5}}{\left(1-q^{5 n-2}\right)^{5}\left(1-q^{5 n-3}\right)^{5}}, q=\exp (2 \pi i \tau) .
$$

Then, we have

$$
g(\tau)=\frac{\frac{1}{2}(-11+5 \sqrt{5}) f(\tau)-1}{-f(\tau)-\frac{1}{2}(-11+5 \sqrt{5})}
$$

Proof. We first set

$$
P_{2}(q):=1+10 \sum_{n=1}^{\infty}\left(d_{2,5}(n)-d_{3,5}(n)\right) q^{n}, Q_{2}(q):=3+10 \sum_{n=1}^{\infty}\left(d_{1,5}(n)-d_{4,5}(n)\right) q^{n},
$$

which implies that

$$
\begin{aligned}
P_{1}(q) & :=\cot \frac{2 \pi}{5}+4 \sin \frac{4 \pi}{5} \sum_{n=1}^{\infty}\left(d_{1,5}(n)-d_{4,5}(n)\right) q^{n}-4 \sin \frac{2 \pi}{5} \sum_{n=1}^{\infty}\left(d_{2,5}(n)-d_{3,5}(n)\right) q^{n}, \\
& =-\frac{2}{5} \sin \frac{2 \pi}{5} P_{2}(q)+\frac{2}{5} \sin \frac{4 \pi}{5} Q_{2}(q), \\
Q_{1}(q) & :=\cot \frac{\pi}{5}+4 \sin \frac{2 \pi}{5} \sum_{n=1}^{\infty}\left(d_{1,5}(n)-d_{4,5}(n)\right) q^{n}+4 \sin \frac{4 \pi}{5} \sum_{n=1}^{\infty}\left(d_{2,5}(n)-d_{3,5}(n)\right) q^{n}, \\
& =\frac{2}{5} \sin \frac{4 \pi}{5} P_{2}(q)+\frac{2}{5} \sin \frac{2 \pi}{5} Q_{2}(q) .
\end{aligned}
$$

The theorem is derived from Corollaries 6.6 and 7.5 . 
Theorem 7.7. For every $\tau \in \mathbb{H}^{2}$, we have

$$
\theta^{\prime}\left[\begin{array}{l}
1 \\
1
\end{array}\right]^{4}=\frac{-(2 \pi)^{4} \zeta_{5}^{4} \theta^{11}\left[\begin{array}{l}
\frac{1}{5} \\
1
\end{array}\right] \theta^{11[}\left[\begin{array}{l}
\frac{3}{5} \\
1
\end{array}\right]}{\theta^{10}\left[\begin{array}{c}
\frac{1}{5} \\
1
\end{array}\right]+11 \theta^{5}\left[\begin{array}{c}
\frac{1}{5} \\
1
\end{array}\right] \theta^{5}\left[\begin{array}{l}
\frac{3}{5} \\
1
\end{array}\right]-\theta^{10}\left[\begin{array}{c}
\frac{3}{5} \\
1
\end{array}\right]},
$$

where $\zeta_{5}=\exp (2 \pi i / 5)$.

Proof. The theorem is derived from formula (6.6) and Theorem 7.4 .

\section{Theorem 7.8.}

(1) For each positive integer $k$, the Eisenstein series $G_{4 k}(\tau)$ can be expressed using a rational expression

$$
\theta\left[\begin{array}{c}
\frac{1}{5} \\
1
\end{array}\right](0, \tau) \text {, and } \theta\left[\begin{array}{c}
\frac{3}{5} \\
1
\end{array}\right](0, \tau) .
$$

(2) For each positive integer $k$, the Eisenstein series $G_{4 k+2}(\tau)$ can be expressed using a rational expression

$$
\theta\left[\begin{array}{c}
\frac{1}{5} \\
1
\end{array}\right](0, \tau), \theta\left[\begin{array}{c}
\frac{3}{5} \\
1
\end{array}\right](0, \tau) \text {, and } \theta^{\prime}\left[\begin{array}{l}
1 \\
1
\end{array}\right](0, \tau) \text {. }
$$

Proof. The theorem can be proved in the same way as Theorem 6.8

\section{Some product-series identities}

\subsection{Some theta functional formulas}

Proposition 8.1. For every $(z, \tau) \in \mathbb{C} \times \mathbb{H}^{2}$, we have

$$
\begin{gathered}
\theta^{2}\left[\begin{array}{c}
1 \\
\frac{3}{5}
\end{array}\right] \theta\left[\begin{array}{l}
1 \\
\frac{1}{5}
\end{array}\right](z) \theta\left[\begin{array}{l}
1 \\
\frac{9}{5}
\end{array}\right](z)-\theta^{2}\left[\begin{array}{l}
1 \\
\frac{1}{5}
\end{array}\right] \theta\left[\begin{array}{l}
1 \\
\frac{3}{5}
\end{array}\right](z) \theta\left[\begin{array}{l}
1 \\
\frac{7}{5}
\end{array}\right](z) \\
+\theta\left[\begin{array}{l}
1 \\
\frac{1}{5}
\end{array}\right] \theta\left[\begin{array}{l}
1 \\
\frac{3}{5}
\end{array}\right] \theta^{2}\left[\begin{array}{l}
1 \\
1
\end{array}\right](z)=0, \\
-\zeta_{5}^{2} \theta^{2}\left[\begin{array}{c}
\frac{3}{5} \\
1
\end{array}\right] \theta\left[\begin{array}{c}
\frac{1}{5} \\
1
\end{array}\right](z) \theta\left[\begin{array}{c}
\frac{9}{5} \\
1
\end{array}\right](z)+\zeta_{5}^{3} \theta^{2}\left[\begin{array}{c}
\frac{1}{5} \\
1
\end{array}\right] \theta\left[\begin{array}{c}
\frac{3}{5} \\
1
\end{array}\right](z) \theta\left[\begin{array}{c}
\frac{7}{5} \\
1
\end{array}\right](z) \\
+\theta\left[\begin{array}{c}
\frac{1}{5} \\
1
\end{array}\right] \theta\left[\begin{array}{c}
\frac{3}{5} \\
1
\end{array}\right] \theta^{2}\left[\begin{array}{l}
1 \\
1
\end{array}\right](z)=0,
\end{gathered}
$$

where $\zeta_{5}=\exp (2 \pi i / 5)$. 
Proof. Here, we provide the proof of equation (8.1). Equation (8.2) can be proved in the same manner. First, note that $\operatorname{dim} \mathcal{F}_{2}\left[\begin{array}{l}0 \\ 0\end{array}\right]=2$, and

$$
\theta\left[\begin{array}{c}
1 \\
\frac{1}{5}
\end{array}\right](z, \tau) \theta\left[\begin{array}{c}
1 \\
\frac{9}{5}
\end{array}\right](z, \tau), \theta\left[\begin{array}{c}
1 \\
\frac{3}{5}
\end{array}\right](z, \tau) \theta\left[\begin{array}{c}
1 \\
\frac{7}{5}
\end{array}\right](z, \tau), \theta^{2}\left[\begin{array}{l}
1 \\
1
\end{array}\right](z, \tau) \in \mathcal{F}_{2}\left[\begin{array}{l}
0 \\
0
\end{array}\right]
$$

Therefore, there exist some complex numbers, $x_{1}, x_{2}$, and $x_{3}$, which are not all zeros, such that

$$
x_{1} \theta\left[\begin{array}{c}
1 \\
\frac{1}{5}
\end{array}\right](z, \tau) \theta\left[\begin{array}{c}
1 \\
\frac{9}{5}
\end{array}\right](z, \tau)+x_{2} \theta\left[\begin{array}{c}
1 \\
\frac{3}{5}
\end{array}\right](z, \tau) \theta\left[\begin{array}{c}
1 \\
\frac{7}{5}
\end{array}\right](z, \tau)+x_{3} \theta^{2}\left[\begin{array}{c}
1 \\
1
\end{array}\right](z, \tau)=0 .
$$

Note that in the fundamental parallelogram, the zero of $\theta\left[\begin{array}{c}1 \\ \frac{1}{5}\end{array}\right](z), \theta\left[\begin{array}{c}1 \\ \frac{3}{5}\end{array}\right](z)$, or $\theta\left[\begin{array}{l}1 \\ 1\end{array}\right](z)$ is $z=2 / 5,1 / 5$ or 0 . By substituting $z=2 / 5,1 / 5$, and 0 , we have

$$
\begin{array}{r}
x_{2} \theta\left[\begin{array}{c}
1 \\
\frac{3}{5}
\end{array}\right]+x_{3} \theta\left[\begin{array}{l}
1 \\
\frac{1}{5}
\end{array}\right]=0, \\
-x_{1} \theta\left[\begin{array}{c}
1 \\
\frac{1}{5}
\end{array}\right] \\
-x_{3} \theta\left[\begin{array}{l}
1 \\
\frac{3}{5}
\end{array}\right]=0, \\
=0 .
\end{array}
$$

By solving this system of equations, we get

$$
\left(x_{1}, x_{2}, x_{3}\right)=\alpha\left(\theta^{2}\left[\begin{array}{c}
1 \\
\frac{3}{5}
\end{array}\right],-\theta^{2}\left[\begin{array}{c}
1 \\
\frac{1}{5}
\end{array}\right], \theta\left[\begin{array}{c}
1 \\
\frac{1}{5}
\end{array}\right] \theta\left[\begin{array}{c}
1 \\
\frac{5}{3}
\end{array}\right]\right) \text { for some } \alpha \in \mathbb{C} \backslash\{0\},
$$

which proves the proposition.

Lemma 8.2. For every $(z, \tau) \in \mathbb{C} \times \mathbb{H}^{2}$, we have

$$
\left\{\frac{\theta^{\prime}\left[\begin{array}{c}
\epsilon \\
\epsilon^{\prime}
\end{array}\right](z)}{\theta\left[\begin{array}{c}
\epsilon \\
\epsilon^{\prime}
\end{array}\right](z)}\right\}^{2}=\frac{\theta^{\prime \prime}\left[\begin{array}{c}
\epsilon \\
\epsilon^{\prime}
\end{array}\right](z)}{\theta\left[\begin{array}{c}
\epsilon \\
\epsilon^{\prime}
\end{array}\right](z)}-\frac{d^{2}}{d z^{2}} \log \theta\left[\begin{array}{c}
\epsilon \\
\epsilon^{\prime}
\end{array}\right](z) .
$$

Proof. The lemma can be proved by direct calculation. 
Theorem 8.3. For every $\tau \in \mathbb{H}^{2}$, we have

$$
\begin{aligned}
\frac{\eta^{5}(\tau)}{\eta(5 \tau)} & =\frac{(q ; q)_{\infty}^{5}}{\left(q^{5} ; q^{5}\right)_{\infty}}=1-5 \sum_{n=1}^{\infty}\left(\sum_{d \mid n} d\left(\frac{d}{5}\right)\right) q^{n}, \\
\frac{\eta^{5}(5 \tau)}{\eta(\tau)} & =q \frac{\left(q^{5} ; q^{5}\right)_{\infty}^{5}}{(q ; q)_{\infty}}=\sum_{n=1}^{\infty}\left(\sum_{d \mid n} \frac{n}{d}\left(\frac{d}{5}\right)\right) q^{n}, \quad q=\exp (2 \pi i \tau),
\end{aligned}
$$

where for each $m \in \mathbb{N}$,

$$
\left(\frac{m}{5}\right)=\left\{\begin{array}{lll}
1 & \text { if } m \equiv \pm 1 & \bmod 5 \\
-1 & \text { if } m \equiv \pm 2 & \bmod 5 \\
0 & \text { if } m \equiv 0 & \bmod 5
\end{array}\right.
$$

Proof. By comparing the coefficients of term $z^{2}$ in equations (8.1) and (8.2), we have

$$
\frac{\left\{\theta^{\prime}\left[\begin{array}{l}
1 \\
1
\end{array}\right]\right\}^{2}}{\theta\left[\begin{array}{c}
1 \\
\frac{1}{5}
\end{array}\right] \theta\left[\begin{array}{c}
1 \\
\frac{3}{5}
\end{array}\right]}=\left.\frac{d^{2}}{d z^{2}} \log \theta\left[\begin{array}{c}
1 \\
\frac{1}{5}
\end{array}\right](z)\right|_{z=0}-\left.\frac{d^{2}}{d z^{2}} \log \theta\left[\begin{array}{c}
1 \\
\frac{3}{5}
\end{array}\right](z)\right|_{z=0}
$$

and

$$
\zeta_{5} \frac{\left\{\theta^{\prime}\left[\begin{array}{l}
1 \\
1
\end{array}\right]\right\}^{2}}{\theta\left[\begin{array}{l}
\frac{1}{5} \\
1
\end{array}\right] \theta\left[\begin{array}{l}
\frac{3}{5} \\
1
\end{array}\right]}=\left.\frac{d^{2}}{d z^{2}} \log \theta\left[\begin{array}{l}
\frac{3}{5} \\
1
\end{array}\right](z)\right|_{z=0}-\left.\frac{d^{2}}{d z^{2}} \log \theta\left[\begin{array}{c}
\frac{1}{5} \\
1
\end{array}\right](z)\right|_{z=0} .
$$

Therefore, the theorem can be obtained using Jacobi's triple product identity (2.5).

Theorem 8.4. For $q \in \mathbb{C}$ with $|q|<1$, set

$$
\begin{aligned}
& P(q)=\cot \frac{2 \pi}{5}+4 \sin \frac{4 \pi}{5} \sum_{n=1}^{\infty}\left(d_{1,5}(n)-d_{4,5}(n)\right) q^{n}-4 \sin \frac{2 \pi}{5} \sum_{n=1}^{\infty}\left(d_{2,5}(n)-d_{3,5}(n)\right) q^{n} \\
& Q(q)=\cot \frac{\pi}{5}+4 \sin \frac{2 \pi}{5} \sum_{n=1}^{\infty}\left(d_{1,5}(n)-d_{4,5}(n)\right) q^{n}+4 \sin \frac{4 \pi}{5} \sum_{n=1}^{\infty}\left(d_{2,5}(n)-d_{3,5}(n)\right) q^{n}
\end{aligned}
$$

Then, we have

$$
\frac{(q ; q)_{\infty}^{5}}{\left(q^{5} ; q^{5}\right)_{\infty}}=-\frac{\sqrt{5}}{4}(3 P-Q)(P+3 Q)
$$


Proof. The theorem follows from equations (6.1) and (8.5).

Theorem 8.5. For $q \in \mathbb{C}$ with $|q|<1$, set

$$
P(q)=1+10 \sum_{n=1}^{\infty}\left(d_{2,5}(n)-d_{3,5}(n)\right) q^{n}, Q(q)=3+10 \sum_{n=1}^{\infty}\left(d_{1,5}(n)-d_{4,5}(n)\right) q^{n}
$$

Then, we have

$$
q \frac{\left(q^{5} ; q^{5}\right)_{\infty}^{5}}{(q ; q)_{\infty}}=-\frac{1}{100}(3 P-Q)(P+3 Q)
$$

and

$$
\frac{(q ; q)_{\infty}^{5}}{\left(q^{5} ; q^{5}\right)_{\infty}}=\frac{1}{4}\left(P^{2}+4 P Q-Q^{2}\right)
$$

Proof. The theorem can be proved in the same way as Theorem 8.4.

\section{Some theorem of Farkas and Kra}

Theorem 9.1. (Farkas and Kra [5, pp. 318]) For every $\tau \in \mathbb{H}^{2}$, we have

$$
\frac{d}{d \tau} \log \left(\frac{\eta(5 \tau)}{\eta(\tau)}\right)+\frac{1}{2 \pi i \cdot 3}\left[\left\{\frac{\theta^{\prime}\left[\begin{array}{c}
1 \\
\frac{1}{5}
\end{array}\right](0, \tau)}{\theta\left[\begin{array}{c}
1 \\
\frac{1}{5}
\end{array}\right](0, \tau)}\right\}^{2}+\left\{\frac{\theta^{\prime}\left[\begin{array}{c}
1 \\
\frac{3}{5}
\end{array}\right](0, \tau)}{\theta\left[\begin{array}{c}
1 \\
\frac{3}{5}
\end{array}\right](0, \tau)}\right\}^{2}\right]=0 .
$$

Proof. Summing both sides of equations (5.7) and (5.8) yields

$$
3 \frac{\theta^{\prime \prime}\left[\begin{array}{c}
1 \\
\frac{1}{5}
\end{array}\right]}{\theta\left[\begin{array}{c}
1 \\
\frac{1}{5}
\end{array}\right]}+3 \frac{\theta^{\prime \prime}\left[\begin{array}{c}
1 \\
\frac{3}{5}
\end{array}\right]}{\theta\left[\begin{array}{c}
1 \\
\frac{3}{5}
\end{array}\right]}-2 \frac{\theta^{\prime \prime \prime}\left[\begin{array}{l}
1 \\
1
\end{array}\right]}{\theta^{\prime}\left[\begin{array}{l}
1 \\
1
\end{array}\right]}+2\left\{\frac{\theta^{\prime}\left[\begin{array}{l}
1 \\
\frac{1}{5}
\end{array}\right]}{\theta\left[\begin{array}{c}
1 \\
\frac{1}{5}
\end{array}\right]}\right\}^{2}+2\left\{\frac{\theta^{\prime}\left[\begin{array}{l}
1 \\
\frac{3}{5}
\end{array}\right]}{\theta\left[\begin{array}{c}
1 \\
\frac{3}{5}
\end{array}\right]}\right\}^{2}=0
$$

The heat equation, Eq. (2.6), implies that

$$
4 \pi i \frac{d}{d \tau} \log \frac{\theta^{3}\left[\begin{array}{c}
1 \\
\frac{1}{5}
\end{array}\right] \theta^{3}\left[\begin{array}{c}
1 \\
\frac{1}{5}
\end{array}\right]}{\left\{\theta^{\prime}\left[\begin{array}{l}
1 \\
1
\end{array}\right]\right\}^{2}}+2\left\{\frac{\theta^{\prime}\left[\begin{array}{c}
1 \\
\frac{1}{5}
\end{array}\right]}{\theta\left[\begin{array}{c}
1 \\
\frac{1}{5}
\end{array}\right]}\right\}^{2}+2\left\{\frac{\theta^{\prime}\left[\begin{array}{c}
1 \\
\frac{3}{5}
\end{array}\right]}{\theta\left[\begin{array}{c}
1 \\
\frac{3}{5}
\end{array}\right]}\right\}^{2}=0 .
$$


Jacobi's triple product identity (2.5) yields

$$
\frac{\theta^{3}\left[\begin{array}{c}
1 \\
\frac{1}{5}
\end{array}\right] \theta^{3}\left[\begin{array}{l}
1 \\
\frac{1}{5}
\end{array}\right]}{\left\{\theta^{\prime}\left[\begin{array}{l}
1 \\
1
\end{array}\right]\right\}^{2}}=\frac{(\sqrt{5})^{3}}{4 \pi^{2}} \frac{\eta^{3}(5 \tau)}{\eta^{3}(\tau)}
$$

which proves the theorem.

Corollary 9.2. For $q \in \mathbb{C}$ with $|q|<1$, set

$$
\begin{aligned}
& P(q)=\cot \frac{2 \pi}{5}+4 \sin \frac{4 \pi}{5} \sum_{n=1}^{\infty}\left(d_{1,5}(n)-d_{4,5}(n)\right) q^{n}-4 \sin \frac{2 \pi}{5} \sum_{n=1}^{\infty}\left(d_{2,5}(n)-d_{3,5}(n)\right) q^{n} \\
& Q(q)=\cot \frac{\pi}{5}+4 \sin \frac{2 \pi}{5} \sum_{n=1}^{\infty}\left(d_{1,5}(n)-d_{4,5}(n)\right) q^{n}+4 \sin \frac{4 \pi}{5} \sum_{n=1}^{\infty}\left(d_{2,5}(n)-d_{3,5}(n)\right) q^{n}
\end{aligned}
$$

Then, we have

$$
P^{2}+Q^{2}=\frac{1}{2}\left\{-E_{2}(q)+5 E_{2}\left(q^{5}\right)\right\} .
$$

Theorem 9.3. For $q \in \mathbb{C}$ with $|q|<1$, set

$$
\begin{aligned}
& P(q)=\cot \frac{2 \pi}{5}+4 \sin \frac{4 \pi}{5} \sum_{n=1}^{\infty}\left(d_{1,5}(n)-d_{4,5}(n)\right) q^{n}-4 \sin \frac{2 \pi}{5} \sum_{n=1}^{\infty}\left(d_{2,5}(n)-d_{3,5}(n)\right) q^{n} \\
& Q(q)=\cot \frac{\pi}{5}+4 \sin \frac{2 \pi}{5} \sum_{n=1}^{\infty}\left(d_{1,5}(n)-d_{4,5}(n)\right) q^{n}+4 \sin \frac{4 \pi}{5} \sum_{n=1}^{\infty}\left(d_{2,5}(n)-d_{3,5}(n)\right) q^{n}
\end{aligned}
$$

Then, we have

$$
\begin{aligned}
& E_{4}\left(q^{5}\right)=P^{4}+3 P^{3} Q-P^{2} Q^{2}-3 P Q^{3}+Q^{4}, \\
& E_{6}\left(q^{5}\right)=\frac{1}{40}\left(P^{2}+Q^{2}\right)\left(41 P^{4}+198 P^{3} Q+154 P^{2} Q^{2}-198 P Q^{3}+41 Q^{4}\right) .
\end{aligned}
$$

Proof. Changing $q \rightarrow q^{5}$ in Ramanujan's system (1.3), we have

$$
q \frac{d}{d q} E_{2}\left(q^{5}\right)=\frac{5}{12}\left\{\left(E_{2}\left(q^{5}\right)\right)^{2}-E_{4}\left(q^{5}\right)\right\}, q \frac{d}{d q} E_{4}\left(q^{5}\right)=\frac{5}{3}\left\{E_{2}\left(q^{5}\right) E_{4}\left(q^{5}\right)-E_{6}\left(q^{5}\right)\right\} .
$$

From Corollary 9.2, we have

$$
E_{2}\left(q^{5}\right)=\frac{1}{5}\left(2 P^{2}+2 Q^{2}+R\right), \quad R=E_{2}(q),
$$


which implies that

$$
q \frac{d}{d q} E_{2}\left(q^{5}\right)=-\frac{7 P^{4}}{20}-\frac{5 P^{3} Q}{4}+\frac{11 P^{2} Q^{2}}{20}+\frac{5 P Q^{3}}{4}-\frac{7 Q^{4}}{20}+\frac{P^{2} R}{15}+\frac{Q^{2} R}{15}+\frac{R^{2}}{60}
$$

Therefore, it follows that

$$
E_{4}\left(q^{5}\right)=\left(E_{2}\left(q^{5}\right)\right)^{2}-\frac{12}{5} q \frac{d}{d q} E_{2}\left(q^{5}\right)=P^{4}+3 P^{3} Q-P^{2} Q^{2}-3 P Q^{3}+Q^{4} .
$$

In the same way, we note that

$$
\begin{gathered}
q \frac{d}{d q} E_{4}\left(q^{5}\right)=-\frac{25 P^{6}}{24}-\frac{25 P^{5} Q}{4}-\frac{65 P^{4} Q^{2}}{8}-\frac{65 P^{2} Q^{4}}{8}+\frac{25 P Q^{5}}{4}-\frac{25 Q^{6}}{24} \\
+P^{3} Q R-\frac{1}{3} P^{2} Q^{2} R-P Q^{3} R+\frac{P^{4} R}{3}+\frac{Q^{4} R}{3}
\end{gathered}
$$

which implies that

$$
\begin{aligned}
E_{6}\left(q^{5}\right) & =E_{2}\left(q^{5}\right) E_{4}\left(q^{5}\right)-\frac{3}{5} q \frac{d}{d q} E_{4}\left(q^{5}\right) \\
& =\frac{1}{40}\left(P^{2}+Q^{2}\right)\left(41 P^{4}+198 P^{3} Q+154 P^{2} Q^{2}-198 P Q^{3}+41 Q^{4}\right) .
\end{aligned}
$$

Corollary 9.4. For $q \in \mathbb{C}$ with $|q|<1$, set

$$
\begin{aligned}
S(q) & =\frac{\sqrt{250-110 \sqrt{5}}}{5} \prod_{n=1}^{\infty} \frac{\left(1-q^{n}\right)^{5}}{\left(1-q^{5 n}\right)^{3}}\left(1+\frac{1-\sqrt{5}}{2} q^{n}+q^{2 n}\right)^{5} \\
& =\frac{\sqrt{250-110 \sqrt{5}}}{5}\left\{1-\frac{5}{2}(1+\sqrt{5}) \sum_{n=1}^{\infty}\left(d_{1,5}(n)-d_{4,5}(n)\right) q^{n}+\frac{5}{2}(7+3 \sqrt{5}) \sum_{n=1}^{\infty}\left(d_{2,5}(n)-d_{3,5}(n)\right) q^{n}\right\} \\
T(q) & =\frac{\sqrt{250+110 \sqrt{5}}}{5} \prod_{n=1}^{\infty} \frac{\left(1-q^{n}\right)^{5}}{\left(1-q^{5 n}\right)^{3}}\left(1+\frac{1+\sqrt{5}}{2} q^{n}+q^{2 n}\right)^{5} \\
& =\frac{\sqrt{250+110 \sqrt{5}}}{5}\left\{1+\frac{5}{2}(-1+\sqrt{5}) \sum_{n=1}^{\infty}\left(d_{1,5}(n)-d_{4,5}(n)\right) q^{n}+\frac{5}{2}(7-3 \sqrt{5}) \sum_{n=1}^{\infty}\left(d_{2,5}(n)-d_{3,5}(n)\right) q^{n}\right\}
\end{aligned}
$$

Then we have

$$
\begin{aligned}
& E_{4}\left(q^{5}\right)=\frac{S^{4}-228 S^{3} T+494 S^{2} T^{2}+228 S T^{3}+T^{4}}{10000} \\
& E_{6}\left(q^{5}\right)=-\frac{\left(S^{2}+T^{2}\right)\left(S^{4}+522 S^{3} T-10006 S^{2} T^{2}-522 S T^{3}+T^{4}\right)}{1000000}
\end{aligned}
$$


and

$$
\begin{aligned}
q^{5}\left(q^{5}: q^{5}\right)_{\infty}^{24} & =\frac{\left(E_{4}\left(q^{5}\right)\right)^{3}-\left(E_{6}\left(q^{5}\right)\right)^{2}}{1728}=-\frac{S T\left(S^{2}+11 S T-T^{2}\right)^{5}}{1000000000000} \\
j\left(q^{5}\right) & =-\frac{\left(S^{4}-228 S^{3} T+494 S^{2} T^{2}+228 S T^{3}+T^{4}\right)^{3}}{S T\left(S^{2}+11 S T-T^{2}\right)^{5}} \\
& =-\frac{\left(f^{4}+228 f^{3}+494 f^{2}-228 f+1\right)^{3}}{f\left(-f^{2}+11 f+1\right)^{5}}, f=T / S .
\end{aligned}
$$

Proof. The corollary can be obtained by considering

$$
S=-3 P+Q, T=P+3 Q \text {, and } f=T / S .
$$

Corollary 9.5. For $q \in \mathbb{C}$ with $|q|<1$, set

$$
\begin{aligned}
V(q) & =\prod_{n=1}^{\infty} \frac{\left(1-q^{n}\right)^{5}}{\left(1-q^{5 n}\right)^{3}}\left(1+\frac{1-\sqrt{5}}{2} q^{n}+q^{2 n}\right)^{5} \\
& =1-\frac{5}{2}(1+\sqrt{5}) \sum_{n=1}^{\infty}\left(d_{1,5}(n)-d_{4,5}(n)\right) q^{n}+\frac{5}{2}(7+3 \sqrt{5}) \sum_{n=1}^{\infty}\left(d_{2,5}(n)-d_{3,5}(n)\right) q^{n}, \\
W(q) & =\prod_{n=1}^{\infty} \frac{\left(1-q^{n}\right)^{5}}{\left(1-q^{5 n}\right)^{3}}\left(1+\frac{1+\sqrt{5}}{2} q^{n}+q^{2 n}\right)^{5} \\
& =1+\frac{5}{2}(-1+\sqrt{5}) \sum_{n=1}^{\infty}\left(d_{1,5}(n)-d_{4,5}(n)\right) q^{n}+\frac{5}{2}(7-3 \sqrt{5}) \sum_{n=1}^{\infty}\left(d_{2,5}(n)-d_{3,5}(n)\right) q^{n} .
\end{aligned}
$$

Then we have

$$
\begin{aligned}
& E_{4}\left(q^{5}\right)=\frac{1}{6250} \times \\
& \times\left\{(123-55 \sqrt{5}) V^{4}+228(11-5 \sqrt{5}) V^{3} W+988 V^{2} W^{2}+228(11+5 \sqrt{5}) V W^{3}+(123+55 \sqrt{5}) W^{4}\right\} \\
& E_{6}\left(q^{5}\right)=-\frac{\left((11 \sqrt{5}-25) V^{2}-(25+11 \sqrt{5}) W^{2}\right)}{1562500} \times \\
& \times\left\{(55 \sqrt{5}-123) V^{4}-522(5 \sqrt{5}-11) V^{3} W+20012 V^{2} W^{2}\right. \\
& \left.\quad+522(11+5 \sqrt{5}) V W^{3}-(123+55 \sqrt{5}) W^{4}\right\}
\end{aligned}
$$


and

$$
\begin{aligned}
q^{5}\left(q^{5} ; q^{5}\right)_{\infty}^{24} & =-\frac{V W(V-W)^{5}}{61035156250} \times \\
\times\{(75025 & \sqrt{5}-167761) V^{5}+10(305 \sqrt{5}-682) V^{4} W+10(5 \sqrt{5}-11) V^{3} W^{2} \\
& \left.+10(11+5 \sqrt{5}) V^{2} W^{3}+10(682+305 \sqrt{5}) V W^{4}+(167761+75025 \sqrt{5}) W^{5}\right\} .
\end{aligned}
$$

Theorem 9.6. For every $\tau \in \mathbb{H}^{2}$, we have

$$
\frac{d}{d \tau} \log \left(\frac{\eta(\tau / 5)}{\eta(\tau)}\right)+\frac{1}{2 \pi i \cdot 3}\left[\left\{\frac{\theta^{\prime}\left[\begin{array}{c}
\frac{1}{5} \\
1
\end{array}\right](0, \tau)}{\theta\left[\begin{array}{c}
\frac{1}{5} \\
1
\end{array}\right](0, \tau)}\right\}^{2}+\left\{\frac{\theta^{\prime}\left[\begin{array}{c}
\frac{3}{5} \\
1
\end{array}\right](0, \tau)}{\theta\left[\begin{array}{c}
\frac{3}{5} \\
1
\end{array}\right](0, \tau)}\right\}^{2}\right]=0
$$

Proof. Summing both sides of equations (5.9) and (5.10) yields

$$
\frac{\theta^{\prime \prime}\left[\begin{array}{l}
\frac{1}{5} \\
1
\end{array}\right]}{\theta\left[\begin{array}{c}
\frac{1}{5} \\
1
\end{array}\right]}+3 \frac{\theta^{\prime \prime}\left[\begin{array}{c}
\frac{3}{5} \\
1
\end{array}\right]}{\theta\left[\begin{array}{c}
\frac{3}{5} \\
1
\end{array}\right]}-2 \frac{\theta^{\prime \prime \prime}\left[\begin{array}{l}
1 \\
1
\end{array}\right]}{\theta^{\prime}\left[\begin{array}{l}
1 \\
1
\end{array}\right]}+2\left\{\frac{\theta^{\prime}\left[\begin{array}{l}
\frac{1}{5} \\
1
\end{array}\right]}{\theta\left[\begin{array}{c}
\frac{1}{5} \\
1
\end{array}\right]}\right\}^{2}+2\left\{\frac{\theta^{\prime}\left[\begin{array}{l}
\frac{3}{5} \\
1
\end{array}\right]}{\theta\left[\begin{array}{c}
\frac{3}{5} \\
1
\end{array}\right]}\right\}^{2}=0 .
$$

The heat equation (2.6) implies that

$$
4 \pi i \frac{d}{d \tau} \log \frac{\theta^{3}\left[\begin{array}{c}
\frac{1}{5} \\
1
\end{array}\right] \theta^{3}\left[\begin{array}{c}
\frac{3}{5} \\
1
\end{array}\right]}{\left\{\theta^{\prime}\left[\begin{array}{l}
1 \\
1
\end{array}\right]\right\}^{2}}+2\left\{\frac{\theta^{\prime}\left[\begin{array}{c}
\frac{1}{5} \\
1
\end{array}\right]}{\theta\left[\begin{array}{c}
\frac{1}{5} \\
1
\end{array}\right]}\right\}^{2}+2\left\{\frac{\theta^{\prime}\left[\begin{array}{c}
\frac{3}{5} \\
1
\end{array}\right]}{\theta\left[\begin{array}{c}
\frac{3}{5} \\
1
\end{array}\right]}\right\}^{2}=0 .
$$

Jacobi's triple product identity (2.5) yields

$$
\frac{\theta^{3}\left[\begin{array}{l}
\frac{1}{5} \\
1
\end{array}\right] \theta^{3}\left[\begin{array}{l}
\frac{3}{5} \\
1
\end{array}\right]}{\left\{\theta^{\prime}\left[\begin{array}{l}
1 \\
1
\end{array}\right]\right\}^{2}}=\frac{\zeta_{5}^{3}}{4 \pi^{2}} \frac{\eta^{3}(\tau / 5)}{\eta^{3}(\tau)}
$$

which proves the theorem.

Corollary 9.7. For $q \in \mathbb{C}$ with $|q|<1$, set

$$
P(q)=1+10 \sum_{n=1}^{\infty}\left(d_{2,5}(n)-d_{3,5}(n)\right) q^{n}, Q(q)=3+10 \sum_{n=1}^{\infty}\left(d_{1,5}(n)-d_{4,5}(n)\right) q^{n} .
$$


Then, we have

$$
P^{2}+Q^{2}=\frac{5}{2}\left\{-E_{2}(q)+5 E_{2}\left(q^{5}\right)\right\} .
$$

Theorem 9.8. For $q \in \mathbb{C}$ with $|q|<1$, set

$$
P(q)=1+10 \sum_{n=1}^{\infty}\left(d_{2,5}(n)-d_{3,5}(n)\right) q^{n}, Q(q)=3+10 \sum_{n=1}^{\infty}\left(d_{1,5}(n)-d_{4,5}(n)\right) q^{n} .
$$

Then, we have

$$
\begin{aligned}
& E_{4}(q)=P^{4}+3 P^{3} Q-P^{2} Q^{2}-3 P Q^{3}+Q^{4}, \\
& E_{6}(q)=-\frac{1}{40}\left(P^{2}+Q^{2}\right)\left(41 P^{4}+198 P^{3} Q+154 P^{2} Q^{2}-198 P Q^{3}+41 Q^{4}\right),
\end{aligned}
$$

and

$$
\begin{aligned}
1728 q(q ; q)_{\infty}^{24} & =\left(E_{4}(q)\right)^{3}-\left(E_{6}(q)\right)^{2}=-\frac{27(3 P-Q)(P+3 Q)\left(P^{2}+4 P Q-Q^{2}\right)^{5}}{1600} \\
j(q) & =\frac{1728\left(E_{4}(q)\right)^{3}}{\left(E_{4}(q)\right)^{3}-\left(E_{6}(q)\right)^{2}}=-\frac{102400\left(P^{4}+3 P^{3} Q-P^{2} Q^{2}-3 P Q^{3}+Q^{4}\right)^{3}}{(3 P-Q)(P+3 Q)\left(P^{2}+4 P Q-Q^{2}\right)^{5}} \\
& =\frac{\left(g^{4}+228 g^{3}+494 g^{2}-228 g+1\right)^{3}}{g\left(g^{2}-11 g-1\right)^{5}}
\end{aligned}
$$

where

$$
g(q)=\frac{3 P-Q}{P+3 Q}=-q \prod_{n=1}^{\infty} \frac{\left(1-q^{5 n-1}\right)^{5}\left(1-q^{5 n-4}\right)^{5}}{\left(1-q^{5 n-2}\right)^{5}\left(1-q^{5 n-3}\right)^{5}} .
$$

Proof. From Corollary 9.7, we have

$$
E_{2}(q)=\frac{-2 P^{2}-2 Q^{2}+25 R}{5}, \quad R=E_{2}\left(q^{5}\right),
$$

which implies that

$$
q \frac{d}{d q} E_{2}(q)=-\frac{7 P^{4}}{100}-\frac{P^{3} Q}{4}+\frac{11 P^{2} Q^{2}}{100}+\frac{P Q^{3}}{4}-\frac{7 Q^{4}}{100}-\frac{P^{2} R}{3}-\frac{Q^{2} R}{3}+\frac{25 R^{2}}{12} .
$$

By Ramanujan's system (1.3), we obtain

$$
E_{4}(q)=\left\{E_{2}(q)\right\}^{2}-12 q \frac{d}{d q} E_{2}(q)=P^{4}+3 P^{3} Q-P^{2} Q^{2}-3 P Q^{3}+Q^{4} .
$$


In the same way, we note that

$$
\begin{aligned}
q \frac{d}{d q} E_{4}(q)=\frac{5 P^{6}}{24}+\frac{5 P^{5} Q}{4} & +\frac{13 P^{4} Q^{2}}{8}+\frac{13 P^{2} Q^{4}}{8}-\frac{5 P Q^{5}}{4}+\frac{5 Q^{6}}{24} \\
- & \frac{5}{3} P^{2} Q^{2} R+5 P^{3} Q R-5 P Q^{3} R+\frac{5 P^{4} R}{3}+\frac{5 Q^{4} R}{3}
\end{aligned}
$$

which implies that

$$
\begin{aligned}
E_{6}(q) & =E_{2}(q) E_{4}(q)-3 q \frac{d}{d q} E_{4}(q) \\
& =-\frac{1}{40}\left(P^{2}+Q^{2}\right)\left(41 P^{4}+198 P^{3} Q+154 P^{2} Q^{2}-198 P Q^{3}+41 Q^{4}\right) .
\end{aligned}
$$

Corollary 9.9. For $q \in \mathbb{C}$ with $|q|<1$, set

$S(q)=q \prod_{n=1}^{\infty} \frac{\left(1-q^{n}\right)^{2}}{\left(1-q^{5 n-2}\right)^{5}\left(1-q^{5 n-3}\right)^{5}}=\sum_{n=1}^{\infty}\left(d_{1,5}(n)-d_{4,5}(n)\right) q^{n}-3 \sum_{n=1}^{\infty}\left(d_{2,5}(n)-d_{3,5}(n)\right) q^{n}$,

and

$T(q)=\prod_{n=1}^{\infty} \frac{\left(1-q^{n}\right)^{2}}{\left(1-q^{5 n-1}\right)^{5}\left(1-q^{5 n-4}\right)^{5}}=1+3 \sum_{n=1}^{\infty}\left(d_{1,5}(n)-d_{4,5}(n)\right) q^{n}+\sum_{n=1}^{\infty}\left(d_{2,5}(n)-d_{3,5}(n)\right) q^{n}$.

Then, we have

$$
\begin{aligned}
& E_{4}(q)=S^{4}-228 S^{3} T+494 S^{2} T^{2}+228 S T^{3}+T^{4} \\
& E_{6}(q)=\left(S^{2}+T^{2}\right)\left(S^{4}+522 S^{3} T-10006 S^{2} T^{2}-522 S T^{3}+T^{4}\right),
\end{aligned}
$$

and

$$
\begin{aligned}
q(q ; q)_{\infty} & =-S T\left(S^{2}+11 S T-T^{2}\right)^{5} \\
j(q) & =-\frac{\left(S^{4}-228 S^{3} T+494 S^{2} T^{2}+228 S T^{3}+T^{4}\right)^{3}}{S T\left(S^{2}+11 S T-T^{2}\right)^{5}}=-\frac{\left(g^{4}-228 g^{3}+494 g^{2}+228 g+1\right)^{3}}{g\left(g^{2}+11 g-1\right)^{5}},
\end{aligned}
$$

where

$$
g=S / T=q \prod_{n=1}^{\infty} \frac{\left(1-q^{5 n-1}\right)^{5}\left(1-q^{5 n-4}\right)^{5}}{\left(1-q^{5 n-2}\right)^{5}\left(1-q^{5 n-3}\right)^{5}}
$$




\section{Remark}

Corollary 9.7 and Theorem 9.8 enable us to consider the extension of the differential fields,

$$
\mathbb{Q}\left(E_{2}(q), E_{4}(q), E_{6}(q)\right) \subset \mathbb{Q}\left(P(q), Q(q), E_{2}(q)\right),
$$

where

$$
P(q)=1+10 \sum_{n=1}^{\infty}\left(d_{2,5}(n)-d_{3,5}(n)\right) q^{n}, Q(q)=3+10 \sum_{n=1}^{\infty}\left(d_{1,5}(n)-d_{4,5}(n)\right) q^{n} .
$$

\section{Riccati equations satisfied by level 5 modular forms}

\subsection{Derivation of Riccati equation (1)}

Proposition 10.1. For every $\tau \in \mathbb{H}^{2}$, we have

$$
\frac{\theta^{\prime \prime}\left[\begin{array}{l}
1 \\
\frac{1}{5}
\end{array}\right]}{\theta\left[\begin{array}{c}
1 \\
\frac{1}{5}
\end{array}\right]}-\frac{\theta^{\prime \prime}\left[\begin{array}{c}
1 \\
\frac{3}{5}
\end{array}\right]}{\theta\left[\begin{array}{c}
1 \\
\frac{3}{5}
\end{array}\right]}=\frac{\left\{\theta^{\prime}\left[\begin{array}{l}
1 \\
1
\end{array}\right]\right\}^{2}}{100 \theta^{6}\left[\begin{array}{c}
1 \\
\frac{1}{5}
\end{array}\right] \theta^{6}\left[\begin{array}{c}
1 \\
\frac{3}{5}
\end{array}\right]}\left\{-8 \theta^{10}\left[\begin{array}{c}
1 \\
\frac{1}{5}
\end{array}\right]+88 \theta^{5}\left[\begin{array}{c}
1 \\
\frac{1}{5}
\end{array}\right] \theta^{5}\left[\begin{array}{c}
1 \\
\frac{3}{5}
\end{array}\right]+8 \theta^{10}\left[\begin{array}{c}
1 \\
\frac{3}{5}
\end{array}\right]\right\} .
$$

Proof. The proposition can be obtained by considering the derivative formulas (5.1) and (5.2) and by comparing the coefficients of term $z^{2}$ in the equation (8.1).

Theorem 10.2. For every $\tau \in \mathbb{H}^{2}$, set

$$
W=\frac{\theta^{5}\left[\begin{array}{c}
1 \\
\frac{1}{5}
\end{array}\right](0, \tau)}{\theta^{5}\left[\begin{array}{l}
1 \\
\frac{3}{5}
\end{array}\right](0, \tau)}=\frac{11+5 \sqrt{5}}{2} \prod_{n=1}^{\infty} \frac{\left(1+\frac{1+\sqrt{5}}{2} q^{n}+q^{2 n}\right)^{5}}{\left(1+\frac{1-\sqrt{5}}{2} q^{n}+q^{2 n}\right)^{5}}, q=\exp (2 \pi i \tau)
$$

$W$ then satisfies the following Riccati equation:

$$
q \frac{d}{d q} W=\frac{1}{(\sqrt{5})^{3}} \frac{(q ; q)_{\infty}^{5}}{\left(q^{5} ; q^{5}\right)_{\infty}}\left(W^{2}-11 W-1\right) .
$$


Proof. The heat equation (2.6) and Proposition 10.1 imply that

$$
\begin{aligned}
& 4 \pi i \frac{d}{d \tau} \log \theta\left[\begin{array}{c}
1 \\
\frac{1}{5}
\end{array}\right]-4 \pi i \frac{d}{d \tau} \log \theta\left[\begin{array}{c}
1 \\
\frac{3}{5}
\end{array}\right] \\
= & \frac{\left\{\theta^{\prime}\left[\begin{array}{c}
1 \\
1
\end{array}\right]\right\}^{2}\left\{-8 \theta^{10}\left[\begin{array}{c}
1 \\
\frac{1}{5}
\end{array}\right]+88 \theta^{5}\left[\begin{array}{c}
1 \\
\frac{1}{5}
\end{array}\right] \theta^{5}\left[\begin{array}{c}
1 \\
\frac{3}{5}
\end{array}\right]+8 \theta^{10}\left[\begin{array}{c}
1 \\
\frac{3}{5}
\end{array}\right]\right\}}{\theta^{5}\left[\begin{array}{l}
1 \\
\frac{1}{5}
\end{array}\right] \theta^{5}\left[\begin{array}{c}
1 \\
\frac{3}{5}
\end{array}\right]},
\end{aligned}
$$

which shows that

$$
\begin{aligned}
& 4 \pi i \frac{d}{d \tau} \log \theta^{5}\left[\begin{array}{c}
1 \\
\frac{1}{5}
\end{array}\right]-4 \pi i \frac{d}{d \tau} \log \theta^{5}\left[\begin{array}{c}
1 \\
\frac{3}{5}
\end{array}\right] \\
= & \frac{\left\{\theta^{\prime}\left[\begin{array}{l}
1 \\
1
\end{array}\right]\right\}^{2}\left\{-8 \theta^{10}\left[\begin{array}{c}
1 \\
\frac{1}{5}
\end{array}\right]+88 \theta^{5}\left[\begin{array}{c}
1 \\
\frac{1}{5}
\end{array}\right] \theta^{5}\left[\begin{array}{c}
1 \\
\frac{3}{5}
\end{array}\right]+8 \theta^{10}\left[\begin{array}{c}
1 \\
\frac{3}{5}
\end{array}\right]\right\}}{\theta^{5}\left[\begin{array}{l}
1 \\
\frac{1}{5}
\end{array}\right] \theta^{5}\left[\begin{array}{c}
1 \\
\frac{3}{5}
\end{array}\right]} .
\end{aligned}
$$

Jacobi's triple product identity (2.5) yields

$$
\frac{\left\{\theta^{\prime}\left[\begin{array}{l}
1 \\
1
\end{array}\right]\right\}^{2}}{\theta\left[\begin{array}{c}
1 \\
\frac{1}{5}
\end{array}\right] \theta\left[\begin{array}{l}
1 \\
\frac{3}{5}
\end{array}\right]}=\frac{4 \pi^{2}}{\sqrt{5}} \prod_{n=1}^{\infty} \frac{\left(1-q^{n}\right)^{5}}{\left(1-q^{5 n}\right)}=\frac{4 \pi^{2}}{\sqrt{5}} \frac{\eta(\tau)^{5}}{\eta(5 \tau)}
$$

By setting

$$
(X, Y)=\left(\theta^{5}\left[\begin{array}{l}
1 \\
\frac{1}{5}
\end{array}\right], \theta^{5}\left[\begin{array}{c}
1 \\
\frac{3}{5}
\end{array}\right]\right)
$$

we obtain

$$
\frac{d X}{d \tau} Y-X \frac{d Y}{d \tau}=\frac{2 \pi i}{(\sqrt{5})^{3}} \frac{\eta^{5}(\tau)}{\eta(5 \tau)}\left(X^{2}-11 X Y-Y^{2}\right),
$$

which implies that

$$
\frac{d}{d \tau} W=\frac{d}{d \tau}\left(\frac{X}{Y}\right)=\frac{\frac{d X}{d \tau} Y-X \frac{d Y}{d \tau}}{Y^{2}}=\frac{2 \pi i}{(\sqrt{5})^{3}} \frac{\eta^{5}(\tau)}{\eta(5 \tau)}\left(W^{2}-11 W-1\right) .
$$

The theorem can be obtained by considering that $\frac{d}{d \tau}=\frac{d q}{d \tau} \frac{d}{d q}=2 \pi i q \frac{d}{d q}$. 


\subsection{Derivation of a Riccati equation (2)}

Proposition 10.3. For every $\tau \in \mathbb{H}^{2}$, we have

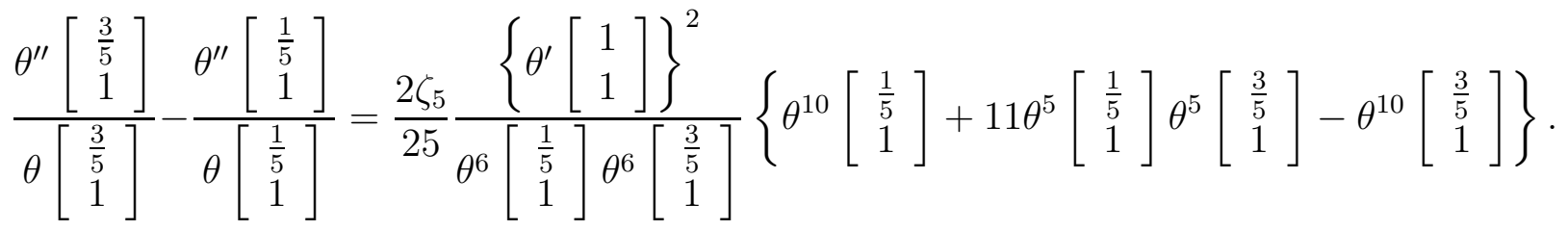

Proof. The proposition can be obtained by considering the derivative formulas (5.3) and (5.4) and by comparing the coefficients of the term $z^{2}$ in equation (8.2).

Theorem 10.4. For every $\tau \in \mathbb{H}^{2}$, set

$$
W=\frac{\theta^{5}\left[\begin{array}{c}
\frac{3}{5} \\
1
\end{array}\right](0,5 \tau)}{\theta^{5}\left[\begin{array}{c}
\frac{1}{5} \\
1
\end{array}\right](0,5 \tau)}=-q \prod_{n=1}^{\infty} \frac{\left(1-q^{5 n-1}\right)^{5}\left(1-q^{5 n-4}\right)^{5}}{\left(1-q^{5 n-2}\right)^{5}\left(1-q^{5 n-3}\right)^{5}}, q=\exp (2 \pi i \tau) .
$$

$W$ then satisfies the following Riccati equation:

$$
q \frac{d}{d q} W=q \frac{\left(q^{5} ; q^{5}\right)_{\infty}^{5}}{(q ; q)_{\infty}}\left(W^{2}-11 W-1\right)
$$

Proof. The heat equation (2.6) and Proposition 10.3 imply that

$$
\begin{aligned}
& 4 \pi i \frac{d}{d \tau} \log \theta\left[\begin{array}{c}
\frac{3}{5} \\
1
\end{array}\right]-4 \pi i \frac{d}{d \tau} \log \theta\left[\begin{array}{c}
\frac{1}{5} \\
1
\end{array}\right]
\end{aligned}
$$

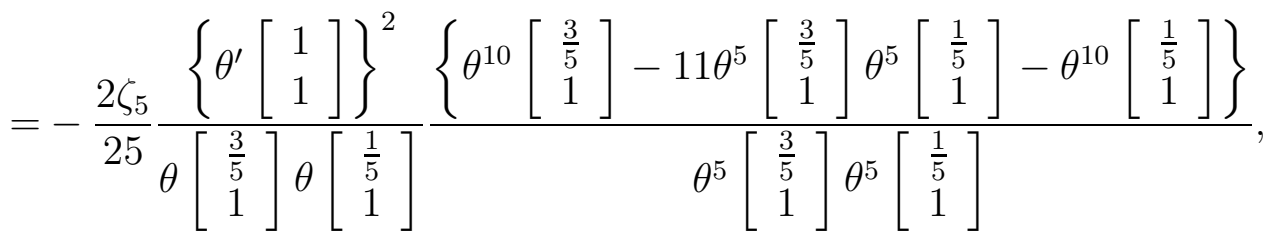

which shows that

$$
\begin{aligned}
& 4 \pi i \frac{d}{d \tau} \log \theta^{5}\left[\begin{array}{c}
\frac{3}{5} \\
1
\end{array}\right]-4 \pi i \frac{d}{d \tau} \log \theta^{5}\left[\begin{array}{c}
\frac{1}{5} \\
1
\end{array}\right]
\end{aligned}
$$

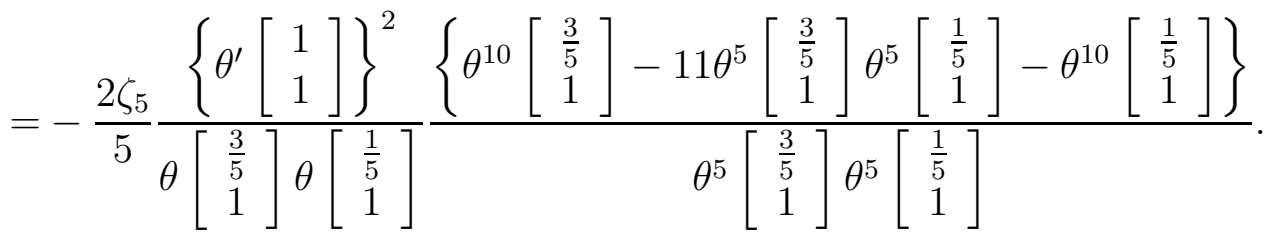


Jacobi's triple product identity (2.5) yields

$$
\frac{\left\{\theta^{\prime}\left[\begin{array}{l}
1 \\
1
\end{array}\right]\right\}^{2}}{\theta\left[\begin{array}{l}
\frac{3}{5} \\
1
\end{array}\right] \theta\left[\begin{array}{l}
\frac{1}{5} \\
1
\end{array}\right]}=\frac{4 \pi^{2}}{\zeta_{5}} y \prod_{n=1}^{\infty} \frac{\left(1-y^{5 n}\right)^{5}}{\left(1-y^{n}\right)}=\frac{4 \pi^{2}}{\zeta_{5}} \frac{\eta^{5}(\tau)}{\eta(\tau / 5)}, y=\exp (2 \pi i \tau / 5)
$$

By setting

$$
(X, Y)=\left(\theta^{5}\left[\begin{array}{c}
\frac{1}{5} \\
1
\end{array}\right](0, \tau), \theta^{5}\left[\begin{array}{c}
\frac{3}{5} \\
1
\end{array}\right](0, \tau)\right)
$$

we obtain

$$
y \frac{d}{d y} W=y \frac{d}{d y}\left(\frac{Y}{X}\right)=y \frac{\left(y^{5} ; y^{5}\right)_{\infty}^{5}}{(y ; y)_{\infty}}\left(W^{2}-11 W-1\right) .
$$

The theorem can be obtained by changing $\tau \longrightarrow 5 \tau$.

\section{References}

[1] T. M. Apostol, Modular functions and Dirichlet series in number theory, Second edition. Grad. Texts in Math. 41 Springer-Verlag, New York, 1990.

[2] J. Chazy, Sur les équations différentielles du troisiéme ordre et dórdre supérieur dont lintgrale générale a ses points critiques fixes, Acta Math. 34 (1911), 317-385.

[3] S. Cooper, Ramanujan's theta functions. Springer, Cham, 2017.

[4] G. Darboux, Sur la théorie des coordonnées curvilignes et les systémes orthogonaux, Ann. Ecole Normale Supérieure 7 (1878) 101-150

[5] H. M. Farkas and I. Kra, Theta constants, Riemann surfaces and the modular group, AMS Grad. Studies in Math. 37 (2001).

[6] G. Halphen, Sur une system déquations differ entielles, C. R. Acad. Sci., Paris 92 (1881), 1101-1103.

[7] C. Hermite, Oeuvres, Vol. II, Gauthier-Villars, Paris, 1908. Note sur la théorie des fonctions elliptiques, pp125-238.

[8] T. Huber, Differential equations for cubic theta functions, Int. J. Number Theory $\mathbf{7 1}$ (2011), 1945-1957. 
[9] K. Matsuda, Differential equations satisfied by $a(q)=\sum_{m, n \in \mathbb{Z}} q^{m^{2}+m n+n^{2}}$, arXiv:1609.07481

[10] Y. Ohyama, Differential relations of theta functions. Osaka J. Math. 32 (1995), 431-450.

[11] S. Ramanujan, On certain arithmetical functions, Trans. Cambridge Philos. Soc. 22, no. 9, (1916) 159-184.

[12] E. T. Whittaker, and G. N. Watson, A course of modern analysis. An introduction to the general theory of infinite processes and of analytic functions; with an account of the principal transcendental functions, Fourth edition. Reprinted Cambridge University Press, New York 1962 